\title{
Lysine acetyltransferase 8 is involved in cerebral development and syndromic intellectual disability
}

\author{
Lin Li, ${ }^{1}$ Mohammad Ghorbani, ${ }^{1}$ Monika Weisz-Hubshman, ${ }^{2,3,4}$ Justine Rousseau, ${ }^{5}$ Isabelle Thiffault, ${ }^{6,7}$ Rhonda E. Schnur, ${ }^{8,9}$ \\ Catherine Breen, ${ }^{10}$ Renske Oegema, ${ }^{11}$ Marjan M.M. Weiss, ${ }^{12}$ Quinten Waisfisz, ${ }^{12}$ Sara Welner, ${ }^{13}$ Helen Kingston, ${ }^{10}$ Jordan A. Hills, ${ }^{14}$ \\ Elles M.J. Boon, ${ }^{12}$ Lina Basel-Salmon, ${ }^{2,3,4,15}$ Osnat Konen, ${ }^{4,16}$ Hadassa Goldberg-Stern, ${ }^{4,17}$ Lily Bazak, ${ }^{3,4}$ Shay Tzur, ${ }^{18,19}$ Jianliang Jin, ${ }^{1,20}$ \\ Xiuli Bi, ${ }^{1}$ Michael Bruccoleri, ${ }^{1}$ Kirsty McWalter, ${ }^{9}$ Megan T. Cho, ${ }^{9}$ Maria Scarano, ${ }^{8} \mathrm{G}$. Bradley Schaefer, ${ }^{14}$ Susan S. Brooks, ${ }^{13}$ \\ Susan Starling Hughes, ${ }^{6,7}$ K.L.I. van Gassen, ${ }^{11}$ Johanna M. van Hagen, ${ }^{12}$ Tej K. Pandita, ${ }^{21}$ Pankaj B. Agrawal, ${ }^{22}$ Philippe M. Campeau, ${ }^{5}$ \\ and Xiang-Jiao Yang ${ }^{1,23}$
}

'Rosalind and Morris Goodman Cancer Research Centre and Department of Medicine, McGill University, Montreal, Quebec, Canada. 2Pediatric Cenetics Unit, Schneider Children's Medical Center of Israel, Petach Tikva, Israel. ${ }^{3}$ Raphael Recanati Genetic Institute, Rabin Medical Center, Petach Tikva, Israel. ${ }^{4}$ Sackler Faculty of Medicine, Tel Aviv University, Tel Aviv, Israel. ${ }^{5}$ Paediatric Department, CHU SainteJustine Hospital, University of Montreal, Quebec, Canada. ${ }^{6}$ Center for Pediatric Cenomic Medicine \& Division of Clinical Genetics, Children's Mercy Hospital, Kansas City, Missouri, USA. ${ }^{7}$ Faculty of Medicine, University of Missouri-Kansas City, Kansas City, Missouri, USA. ${ }^{8}$ Division of Cenetics, Cooper University Health Care, Camden, New Jersey, USA. ${ }^{9}$ CeneDx, Gaithersburg, Maryland, USA. ${ }^{10}$ Manchester Centre for Cenomic Medicine, Central Manchester University Hospitals NHS Foundation Trust, Saint Mary's Hospital, Manchester, United Kingdom. "Department of Cenetics, University Medical Center Utrecht, Utrecht, Netherlands. ${ }^{2}$ Department of Clinical Genetics, Amsterdam University Medical Center, Amsterdam, Netherlands. ${ }^{13}$ Division of Pediatric Medical Genetics, The State University of New Jersey, Rutgers Robert Wood Johnson Medical School, New Brunswick, New Jersey, USA. ${ }^{14}$ University of Arkansas for Medical Sciences, Little Rock, Arkansas, USA. ${ }^{15}$ Felsenstein Medical Research Center, Rabin Medical Center, Petach Tikva, Israel. ${ }^{16 I}$ Imaging Department, Schneider Children's Medical Center of Israel, Petach Tikva, Israel. " ${ }^{17}$ Epilepsy Unit and EEG Laboratory, Schneider Medical Center, Petach Tikva, Israel. . ${ }^{18}$ Laboratory of Molecular Medicine, Rappaport Faculty of Medicine and Research Institute, Technion-Israel Institute of Technology, Haifa, Israel. ${ }^{19}$ Cenomic Research Department, Emedgene Technologies, Tel Aviv, Israel ${ }^{20}$ Research Center for Bone and Stem Cells, Department of Human Anatomy, Key Laboratory of Aging \& Disease, Nanjing Medical University, Nanjing, Jiangsu, China. ${ }^{21}$ Department of Radiation Oncology, Houston Methodist Research Institute, Houston, Texas, USA. ${ }^{22}$ Divisions of Newborn Medicine and Genetics and Genomics, The Manton Center for Orphan Disease Research, Boston Children's Hospital, Harvard Medical School, Boston, Massachusetts, USA. ${ }^{23}$ Departments of Biochemistry and Medicine, McGill University Health Center, Montreal, Quebec, Canada.

Epigenetic integrity is critical for many eukaryotic cellular processes. An important question is how different epigenetic regulators control development and influence disease. Lysine acetyltransferase 8 (KAT8) is critical for acetylation of histone $\mathrm{H} 4$ at lysine 16 (H4K16), an evolutionarily conserved epigenetic mark. It is unclear what roles KAT8 plays in cerebral development and human disease. Here, we report that cerebrum-specific knockout mice displayed cerebral hypoplasia in the neocortex and hippocampus, along with improper neural stem and progenitor cell (NSPC) development. Mutant cerebrocortical neuroepithelia exhibited faulty proliferation, aberrant neurogenesis, massive apoptosis, and scant H4K16 propionylation. Mutant NSPCs formed poor neurospheres, and pharmacological KAT8 inhibition abolished neurosphere formation. Moreover, we describe KAT8 variants in 9 patients with intellectual disability, seizures, autism, dysmorphisms, and other anomalies. The variants altered chromobarrel and catalytic domains of KAT8, thereby impairing nucleosomal H4K16 acetylation. Valproate was effective for treating epilepsy in at least 2 of the individuals. This study uncovers a critical role of KAT8 in cerebral and NSPC development, identifies 9 individuals with KAT8 variants, and links deficient H4K16 acylation directly to intellectual disability, epilepsy, and other developmental anomalies.

\section{Introduction}

Hundreds of epigenetic regulators have been identified and characterized, but much less is known about their pathophysiological roles. Lysine acetyltransferases (KATs) form a prominent group of chromatin regulators, catalyze reversible $\mathrm{N}^{\varepsilon}$-acetylation of histones and other proteins, and generate dynamic $\mathrm{N}^{\varepsilon}$-acetylomes in response to signaling cues $(1,2)$. The human genome encodes

Authorship note: LL, MG, and MWH contributed equally to this work. Conflict of interest: RES, MTC, and KM are affiliated with GeneDx, Inc., a wholly owned subsidiary of OPKO Health, Inc., USA.

Copyright: (5) 2020, American Society for Clinical Investigation.

Submitted: June 18, 2019; Accepted: November 21, 2019; Published: February 4, 2020

Reference information: / Clin Invest. 2020;130(3):1431-1445.

https://doi.org/10.1172/JCI131145
15 KATs, grouped into 3 major families: general control nonderepressible 5 and its related proteins, CREB-binding protein (CBP) and its paralog (p300), and the Moz, Ybf2/Sas3, Sas2, and Tip60 (MYST) family $(1,2)$. Within the latter, there are 5 members, including KAT5, KAT6A, KAT6B, KAT7, and KAT8. Despite their conserved MYST domains for intrinsic acetyltransferase activity $(1,2)$, these enzymes show site specificity toward histones and inscribe different epigenetic marks in vivo. KAT7 is critical for histone $\mathrm{H} 3$ acetylation at lysine 14 (3), whereas KAT6A and KAT6B are responsible for acetylation at lysine $23(4,5)$. KAT8 acetylates histone $\mathrm{H} 4$ at lysine (K) 16 (H4K16) (6-9), indicating that this enzyme is functionally different from other MYST family members $(10,11) . K A T 6 A$ and $K A T 6 B$ pathogenic variants are linked to syndromic intellectual disability (11), but it remains unclear whether any other MYST proteins are altered in genetic diseases. 
Like Drosophila Mof (critical for gene dosage compensation in male flies) (12-14), KAT8 is essential for H4K16 acetylation in mammals $(7,9)$ and critical for DNA damage responses and nuclear architecture $(9,15-18)$. Its loss causes cell cycle arrest, senescence, and apoptosis $(9,17,19)$. Biological functions of KAT8 have been investigated by gene disruption in mice. Global loss of Kat 8 arrests mouse embryogenesis at the implantation (16) or gastrulation stage (7). Through conditional deletion, mouse Kat 8 has been shown to be important for oocyte development (20), Purkinje cell maintenance (21), cardiomyocyte mitochondrial respiration (22), hematopoiesis $(23,24)$, and antiviral immunity (25). However, it remains unclear whether KAT8 has any roles in cerebral development. To address this, we inactivated the mouse gene in the cerebrum and its embryonic primordium. The mutant pups displayed early lethality and severe cerebral hypoplasia, along with defective NSPC development. Consistent with these murine results, we have identified KAT8 variants in 9 human individuals who exhibit syndromic intellectual disability and brain abnormalities. Moreover, these variants are defective in promoting H4K16 acetylation in vitro. This study thus uncovers an essential role of mouse Kat8 in cerebral development, identifies human subjects with KAT8 variants, and links this unique epigenetic modifier to cerebral and intellectual development.

\section{Results}

Cerebrum-specific deletion of mouse Kat8 causes early lethality and cerebral hypoplasia. To investigate whether KAT8 is important for cerebral development, we took a mouse genetic approach. For this, we first analyzed distribution of H4K16 acetylation (H4K16ac) during mouse cerebral development. As shown in Supplemental Figure 1A (supplemental material available online with this article; https://doi.org/10.1172/JCI131145DS1), the H4K16ac level was high in the neocortex and hippocampus of the adult brain, as well as in these areas at P5 and in the cerebrocortical neuroepithelium at E12.5. This distribution pattern suggests an important role of KAT8, the major enzyme responsible for $\mathrm{H} 4 \mathrm{~K} 16 \mathrm{ac}(6,7,9)$, during cerebral development. Related to this, RNA-Seq revealed that Kat8 and the genes of KAT8-associated subunits are well transcribed in the neonatal cerebrum (Supplemental Figure 1B) and embryonic neurospheres (Supplemental Figure 1C), suggesting the importance of KAT8 during cerebral development.

To evaluate this link directly, we generated cerebrum-specific knockout mice by using the Emx1-Cre strain, which expresses the Cre recombinase specifically in the cerebrum and its embryonic precursor starting at E10.5 $(26,27)$. This Cre line was mated with $\mathrm{Kat}^{f / / f l}$ mice (21), to produce Kat $8^{f / f l}$ Emx1-Cre-knockout (or $\mathrm{cKO}$ ) mice. The knockout pups were born at a normal Mendelian ratio and appeared grossly normal. In week 1 , visual inspection revealed no obvious abnormality in the gross appearance of homozygous mutant pups, except some were slightly smaller than wild-type littermates (Supplemental Figure 2A). However, starting from the middle of week 2, they failed to thrive and became much smaller than the wild-type (Figure 1A and Supplemental Figure 2B). They were hyperactive and easily irritated. In week 3 , a majority of them became runty (Figure 1, A and B) and subsequently died (or required euthanizing because of poor health). Thus, the homozygous mutant pups exhibited postnatal growth retardation and preweaning lethality.
Comparison of 3-week-old wild-type and mutant mice led to recognition of a "flat-head" phenotype, i.e., flatter surface above the mutant frontal skull than the wild-type counterpart (Figure 1C). Autopsy revealed a much smaller mutant cerebrum (Figure 1D). After recognizing this phenotype, we visually inspected younger pups and could identify homozygous mutant pups with this feature even at P6 (Supplemental Figure 2A). We next dissected out and compared the wild-type and mutant brains. As shown in Figure 1E, the mutant cerebral cortex was largely missing. The defect was also evident in the mutant at P5 (Figure $1 \mathrm{~F}$ and Supplemental Figure 2C) and P1 (Supplemental Figure 2D). Quantification revealed that the mutant brain was significantly smaller than the wild-type, starting at P1 (Figure 1G). Interestingly, the defects could be traced back to the embryonic stage as early as E16.5 (Supplemental Figure 2, E and F). Thus, cerebrum-specific deletion of Kat8 causes severe cerebral hypoplasia, starting at embryonic stages.

We next analyzed the impact of Kat8 deletion on H4K16ac. Immunofluorescence microscopy was used to detect this modification in embryonic sections. As shown in Supplemental Figure 1D, H4K16ac disappeared in the cerebrocortical neuroepithelium and hippocampal primordium at E12.5, but not the in the medial or lateral ganglionic eminence, indicating that the Emx1-Cre line induces Kat8 deletion and abolishes H4K16 acetylation in the cerebrocortical neuroepithelium.

Kat8 deletion leads to cerebral defects and premature neurogenesis. To examine the cerebral defects further, we performed histological analysis of wild-type and mutant brain or embryo sections with Nissl staining. As shown in Figure 1, H-K, cerebral morphology was altered in the neocortex and hippocampus (or their developmental precursors) of the mutant brain, starting at E13.5. In contrast, such alteration was not so obvious at E12.5 (Figure 1L). To substantiate these histological observations, we carried out immunostaining to examine defects at the molecular and cellular levels. For this, we used various antibodies specific to cortical lamination markers, such as CUT-like homeobox 1 (CUX1) and chicken ovalbumin upstream promoter transcription factor-interacting protein 2 (CTIP2), to stain different layers of the neocortex as described previously (28). Even at E16.5, CTIP2 ${ }^{+}$ cells were largely missing in the mutant cerebral cortex whereas such cells were found to form a layer at the normally expected position in the wild-type cortex (Supplemental Figure 3). Moreover, $\mathrm{CUX} 1^{+}$cells were also largely missing in the mutant cerebral cortex (Supplemental Figure 3). These results indicate that neocortical lamination was altered in the mutant brain, starting at a prenatal stage, as early as E16.5.

We also tested Tuj1, an antibody recognizing a neuronspecific $\beta$-tubulin, to stain embryonic sections at earlier developmental time points. Because the morphological defects in the mutant cerebrocortical epithelium were obvious from Nissl staining at E13.5 (Figure 1K) but not E12.5 (Figure 1L), we analyzed embryonic sections at these 2 stages. As shown in Figure 2A, there were many more neurons in the mutant cerebrocortical neuroepithelium and hippocampal primordium at E13.5. Moreover, Tuj $1^{+}$neurons formed a discrete layer at the outer edge of the wildtype cerebrocortical neuroepithelium; the distribution of such neurons was much broader in the mutant, indicative of premature 
A

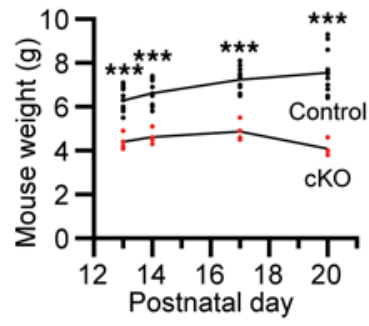

B

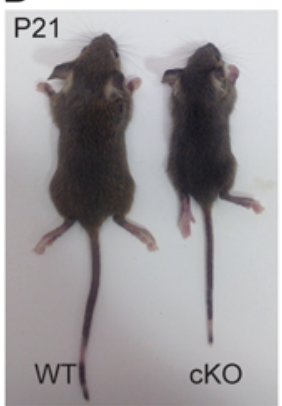

C

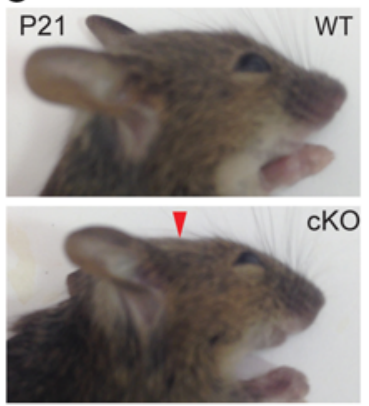

G

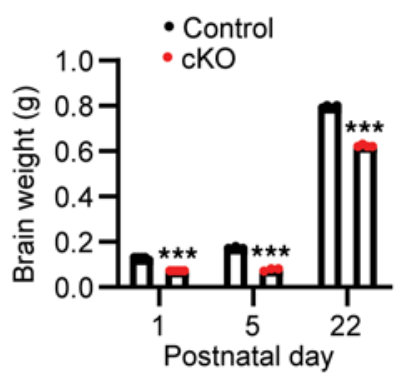

D

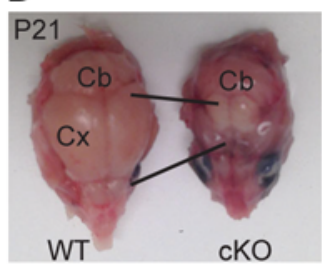

\section{E}

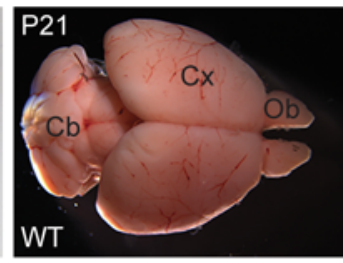

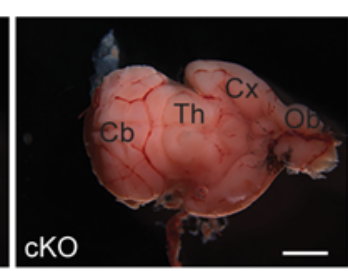
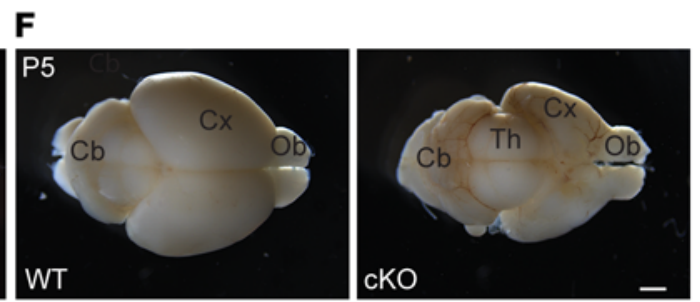

H

P22

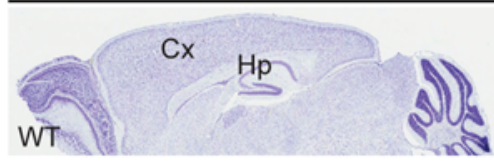

I

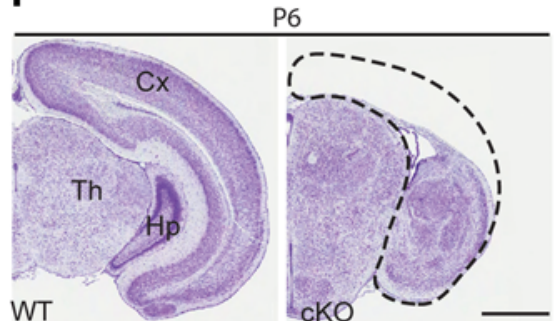

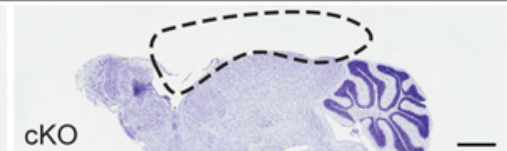

J

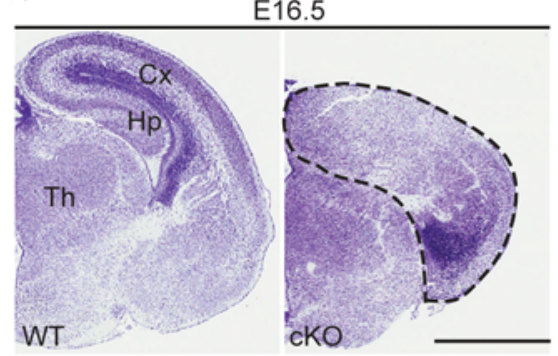

$\mathbf{K}$

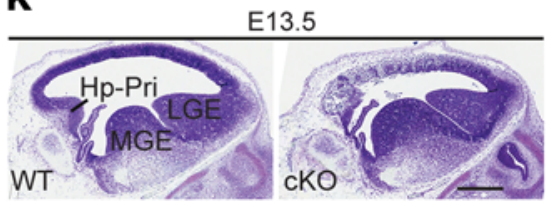

$\mathbf{L}$

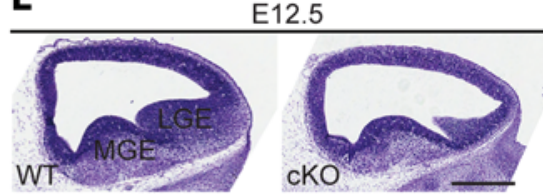

Figure 1. Conditional Kat8 deletion causes early lethality and cerebral hypoplasia. (A) Growth curves for control and homozygous knockout (cKO) mice ( $n=14$ and 4, respectively). (B) Photos of wild-type and cKO mice at P21. (C) Enlarged photos of head parts of the mice shown in B. The "flat-head" phenotype refers to the flat head surface above the cerebrum (indicated with a red arrowhead). (D) Photos of deskinned heads from the mice shown in B. (E) Brain images for the wild-type and cKO mice shown in B. (F) Representative brain images for the wild-type and cKO mice at P5. See Supplemental Figure 2, C-F, for brain images of another pair at P5 and 3 pairs at P1, E18.5, and E16.5. (C) Brain weight at P1, P5, and P22 ( $n=5,3$, and 4 for each genotype, respectively). (H and I) Nissl staining of sagittal (H) or coronal (I) brain sections at P22 or P6. (J-L) Nissl staining of coronal (J) or sagittal (K-L) embryo sections at E16.5, E13.5, or E12.5. For panels H-L, mainly the cerebrum or its precursor is shown. Dashed lines demarcate the cerebral cortex. The mutant cortex is largely lost at P6 (I) and P22 (H). Neocortical and hippocampal lamination is not evident in the mutant at E16.5 (J). The small mutant LGE at E12.5 is due to section orientation. For (B-F), images are representative of 5 pairs of wild-type and mutant mice, and for (H-L), each image is representative of 3 experiments. For $\mathbf{A}$ and $\mathbf{G}$, unpaired 2-tailed Student's $t$ tests were used; ${ }^{* * *} P<0.001$. Scale bars: $2 \mathrm{~mm}(\mathbf{E}), 1 \mathrm{~mm}(\mathbf{F}$ and $\mathbf{H}-\mathbf{J})$, and $0.5 \mathrm{~mm}(\mathbf{K}-\mathbf{L})$. Cb, cerebellum; CP, cortical plate; Cx, cerebral cortex; Hp, hippocampus; Hp-Pri, hippocampal primordium; LGE, lateral ganglionic eminence; MCE, medial ganglionic eminence; Ob, olfactory bulb; Th, thalamus.

neuronal differentiation and/or aberrant neuronal migration in the mutant. In contrast, such abnormalities were not so obvious at E12.5 (Figure 2B). Because H4K16ac disappeared in the mutant cerebrocortical neuroepithelia at E12.5 (Supplemental Figure 1D), the lack of obvious defects with Tuj1 ${ }^{+}$neurons at E12.5 was not due to Kat8 deletion efficiency. Thus, cerebrum-specific Kat8 deletion leads to cerebrocortical neuroepithelial defects and promotes aberrant neurogenesis, starting at E13.5.
Kat8 deletion arrests development of NSPCs. Nissl staining revealed abnormal cerebrocortical neuroepithelia and hippocampal primordia in mutant embryos at E13.5 (Figure 1K) but not E12.5 (Figure 1L). NSPC precursors, such as radial glia cells, reside in the ventricular/subventricular zones (29). To investigate whether these cells are affected, we carried out immunostaining of embryonic and brain sections using an antibody against sex-determining region Y-related high-mobility-group 

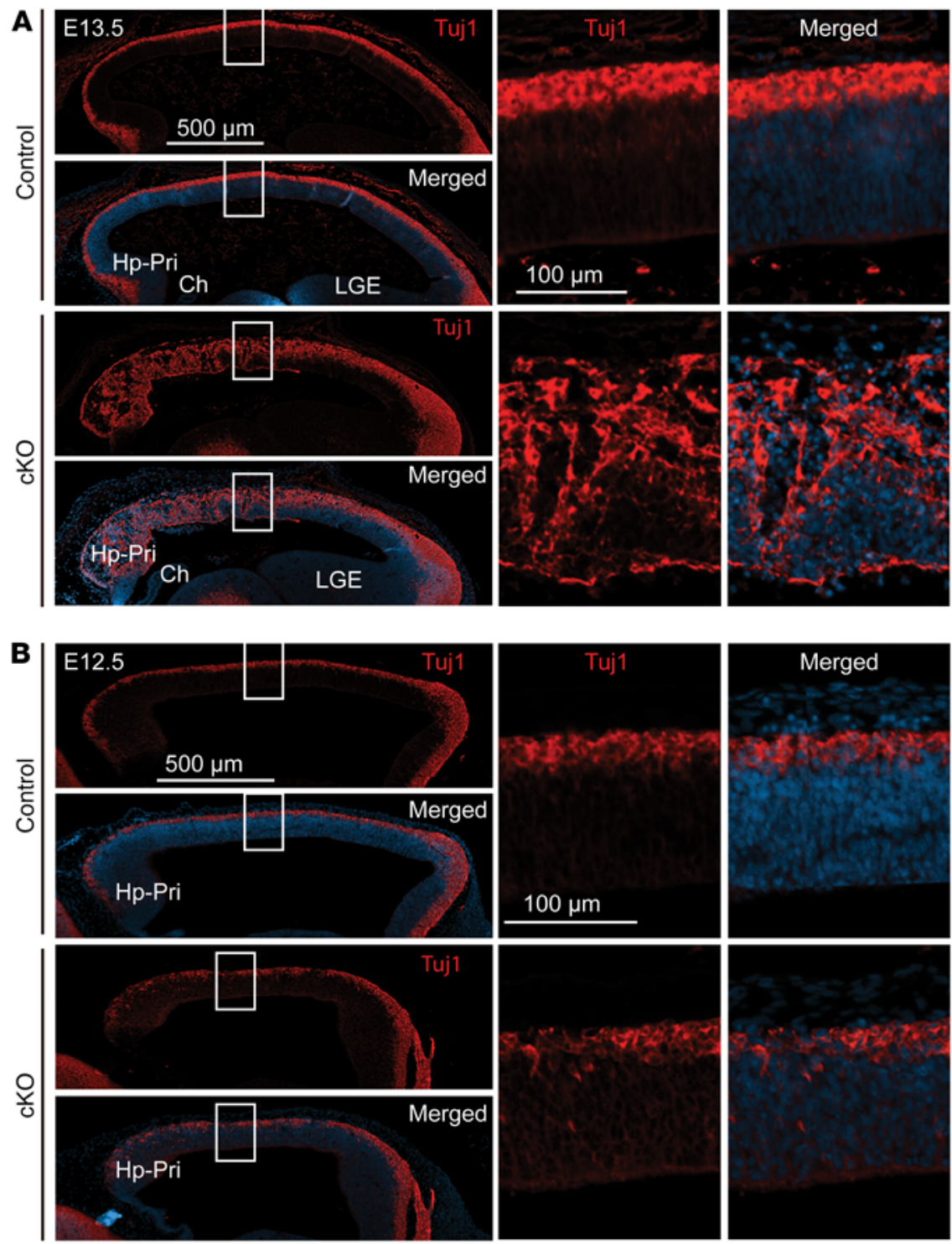

Figure 2. Kat8 deletion promotes neurogenesis at E13.5. (A) Immunostaining analysis of E13.5 brain sections uncovered many more Tuj1+ neurons in the mutant precortical plate. (B) Immunostaining analysis of E12.5 brain sections with similar distribution of Tuj $1^{+}$neurons between the wild-type and mutant precortical plates. The images are representative of 3 experiments. Only the precortical plates are shown here. Scale bars: $500 \mu \mathrm{m}$ (left), $100 \mu \mathrm{m}$ (middle and right).

and mutant cerebrocortical neuroepithelia at E12.5 (Supplemental Figure 4B), indicating that Kat8 is not required for formation of the $\mathrm{TBR} 2^{+}$progenitor cell layer at this stage. Thus, Kat8 loss reduces the number of NSPCs available for cerebrocortical development, starting at E13.5.

Kat8 deletion inhibits cell proliferation and triggers massive apoptosis. To understand the cellular mechanisms underlying NSPC loss, we investigated how Kat8 deletion affects different cellular programs. For this, we first analyzed the impact on cell proliferation, by 1-hour BrdU labeling of E13.5 embryos in vivo. As shown in Figure 4, there were significantly fewer $\mathrm{BrdU}^{+}$or $\mathrm{Ki}-67^{+}$cells in the mutant cerebrocortical neuroepithelium than the wild-type counterpart, indicating that Kat8 deletion inhibits cell proliferation. In stark contrast, the $\mathrm{BrdU}^{+}$or $\mathrm{Ki}-67^{+}$cell number remained comparable between the wild-type and mutant cerebrocortical neuroepithelium at E12.5 (Supplemental Figure 5A), indicating that Kat8 is not essential for cell proliferation at this embryonic stage.

Kat8 is critical for DNA damage responses and nuclear architecture (9, 15-17), so we investigated the impact of Kat8 deletion on DNA damage responses by immunostaining with an anti-phosphoSer139 H2A.X ( $\gamma$ H2A.X) antibody. As shown in Supplemental Figure 5B, there was no obvious difference between the wild-type and mutant cerebrocortical neuroepithelia in terms of $\gamma \mathrm{H} 2 \mathrm{~A} . \mathrm{X}^{+}$cells, suggesting that Kat8 deletion exerts minimal effects on DNA damage response under the uninduced conditions in vivo. We next analyzed the impact of Kat8 deletion on apoptosis. For this, we carried out TUNEL assays. As shown in Figure 5A, there were many TUNEL ${ }^{+}$cells in the mutant cerebrocortical

box 2 (SOX2), an NSPC-specific marker (30). At E13.5, there were dramatically fewer SOX $2^{+}$cells in the mutant cerebrocortical neuroepithelium and hippocampal primordium than the control (Figure 3A), whereas the number of these cells was not obviously affected in the mutant at E12.5 (Figure 3B), indicating that Kat8 is essential for $\mathrm{SOX}_{2}{ }^{+}$cell layer formation at E13.5 but not E12.5. We next analyzed embryonic and brain sections using an antibody specific to the transcription factor T-box brain protein 2 (TBR2), a marker of neuronal progenitor cells important for hippocampus development $(31,32)$. As shown in Supplemental Figure $4 \mathrm{~A}$, the $\mathrm{TBR} 2^{+}$progenitor number was reduced profoundly in mutant cerebrocortical neuroepithelia at E13.5, indicating that Kat8 deletion exhausts the neural progenitor cell population at this stage of development. In stark contrast, the TBR2+ progenitor cell number remained comparable between the wild-type neuroepithelia at E13.5, indicating that Kat8 deletion promotes apoptosis. Consistent with this, immunostaining with the anticleaved caspase- 3 antibody uncovered caspase- 3 activation in the mutant (Figure 5B). Moreover, the $\mathrm{TUNEL}^{+}$cell number also increased dramatically in the mutant cerebrocortical neuroepithelium and hippocampal primordium even at E12.5 (Supplemental Figure 6A). Similarly, at this stage, many mutant cells were positive for activated caspase-3 (Supplemental Figure 6B), indicating that Kat8 deletion also promotes apoptosis at E12.5. Together, the above assays indicate that Kat8 deletion impairs cell proliferation but stimulates apoptosis, starting at E12.5.

KAT8 is required for neurosphere formation in vitro. To investigate whether mutant NSPCs are still functional, we adopted neurosphere formation assays, which have been widely used to examine NSPC potential in vitro (33). For these assays, we col- 

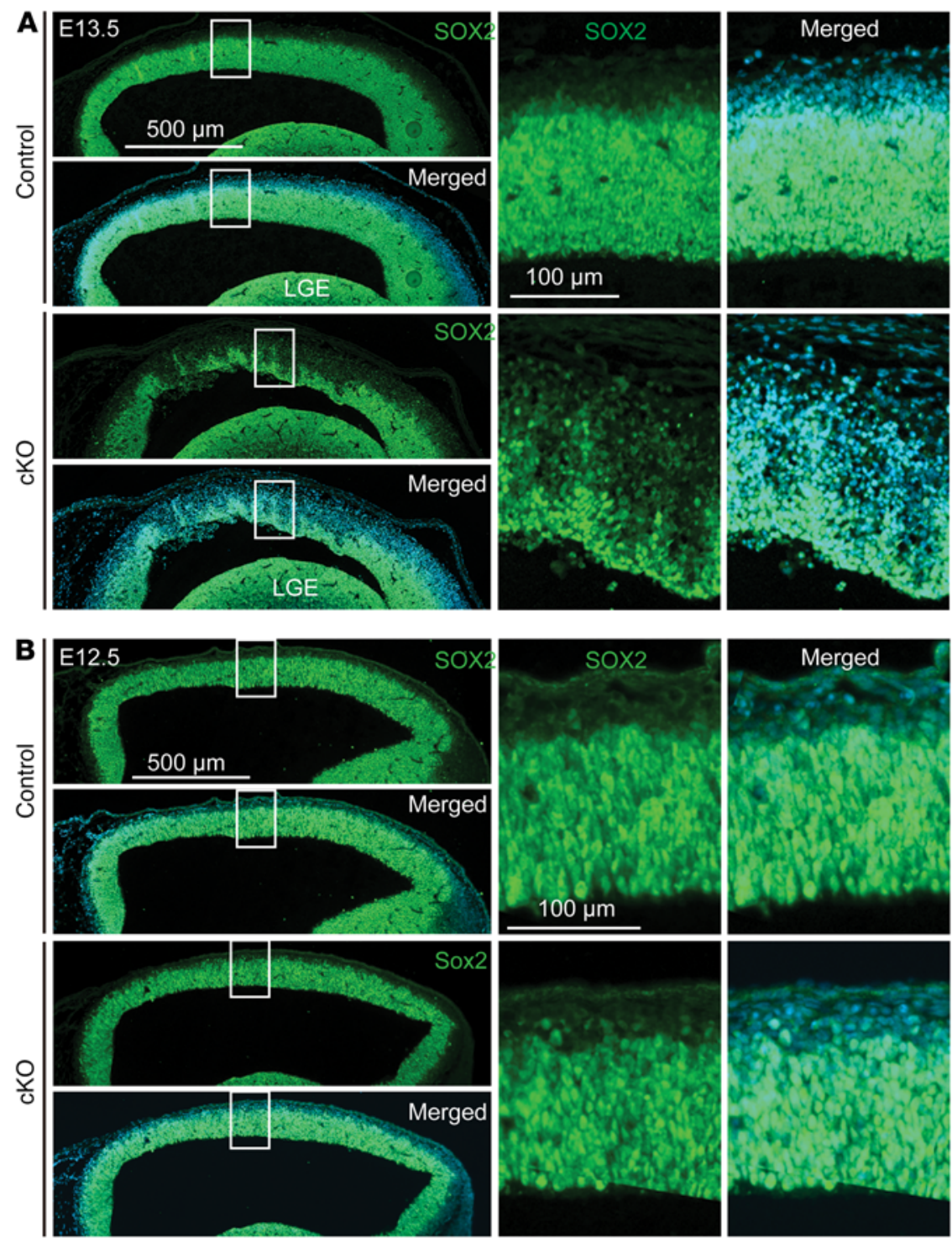

Figure 3. Cerebrum-specific Kat8 deletion reduces the embryonic NSPC population. (A) Immunostaining analysis of E13.5 control and mutant embryonic sections with an anti-SOX2 antibody. Enlarged images of the squared areas are shown at the middle and right. (B) Same as $\mathbf{A}$ except that E12.5 embryonic sections were analyzed. The images are representative of 4 (A) or 3 (B) experiments. Scale bars: $500 \mu \mathrm{m}$ (left), $100 \mu \mathrm{m}$ (middle and right).

lected cerebral cortices from wild-type and mutant embryos at E16.5 to prepare single-cell suspensions for subsequent neurosphere formation in vitro in an NSPC culture medium. As shown in Figure 6A, wild-type but not mutant single cells formed round neurospheres during the first 4 days of culturing, indicating that cerebrum-specific Kat8 deletion impairs neurosphere formation in vitro. We next took a pharmacological approach to substantiate this conclusion. No KAT8-specific inhibitors have been developed yet. To circumvent this, we used 2 selective inhibitors, MG149 and NU9056. The former targets both KAT5 and KAT8 (34), whereas NU9056 is specific to KAT5 (35). The difference between these 2 inhibitors would allow us to compare their impact and deduce the effect of KAT8 inhibition on neurospheres. Single-cell suspensions were prepared from wild-type E16.5 embryonic cerebral cortices for neurosphere formation in vitro in the presence or absence of the inhibitors. As shown in Figure 6B, MG149 exerted much more dramatic effects than NU9056, supporting the importance of KAT8 in neurosphere formation in vitro.

KAT8 is critical for histone H4K16 propionylation in vivo. In addition to H4K16 acetylation, KAT8 promotes histone propionylation in vitro (36), but it is unclear which lysine residue is propionylated, nor was the physiological relevance investigated. KAT8 is an H4K16 acetyltransferase $(6,7,9)$, so we postulated that it is also required for H4K16 propionylation (H4K16pr). To test this, we carried out immunofluorescence microscopy to detect H4K16pr in wild-type and mutant embryonic sections. As shown in Figure 6C, this novel acylation was detectable in the wild-type cerebrocortical neuroepithelium and hippocampal primordium. Its subnuclear distribution appeared more uniform than H4K16ac (Supplemental Figure 7, A and B). In the mutant, H4K16pr virtually disappeared in these 2 areas (Figure 6D). This difference was also obvious between the wild-type and mutant cerebrocortical neuroepithelia at E12.5 (Supplemental Figure 7, C and D). In contrast, the modification was not altered in the mutant ganglionic eminence (Figure 6, C and D, and Supplemental Figure 7, C and D), where the Emx1-Cre transgene is not expressed $(26,27)$. These results indicate that Emx1-Cre-mediated deletion of Kat8 depletes H4K16pr in the cerebrocortical neuroepithelium and hippocampal primordium.

Identification of individuals with intellectual disability harboring KAT8 variants. No direct disease links to KAT8 have been reported, so we sought to identify patients possessing its variants. For this, we leveraged exomesequencing projects carried out around the world. In addition to use of prior collaborations, we employed Web-based matching tools such as Genematcher (37) and Database of Chromosomal Imbalance and Phenotype in Humans Using Ensembl Resources (DECIPHER) (38) to identify individuals with KAT8 variants. As a result, we identified 9 unrelated cases (Figure 7A and Supplemental Table 1), with 8 (individuals T1-T8) carrying heterozygous de novo variants and 1 (individual T9) harboring biallelic variants. Coincidentally, 3 unrelated probands (T1, T2, and T3, Figure 7A and Supplemental Table 1) shared a recurrent de novo variant. Individual T9 inherited biallelic variants from her asymptomatic parents (Supplemental Table 1). Her sister carried 1 such variant and showed no obvious symptoms (Supplemental Table 1). One explanation for the asymptomatic phenotypes in her and the parents is incomplete genetic penetrance of the 2 variants. Of relevance, incomplete penetrance of heterozygous variants occurs with another epigenetic modifier $(5,39)$, and 1 LMNA variant causes phenotypes in some but not other carriers (40). 

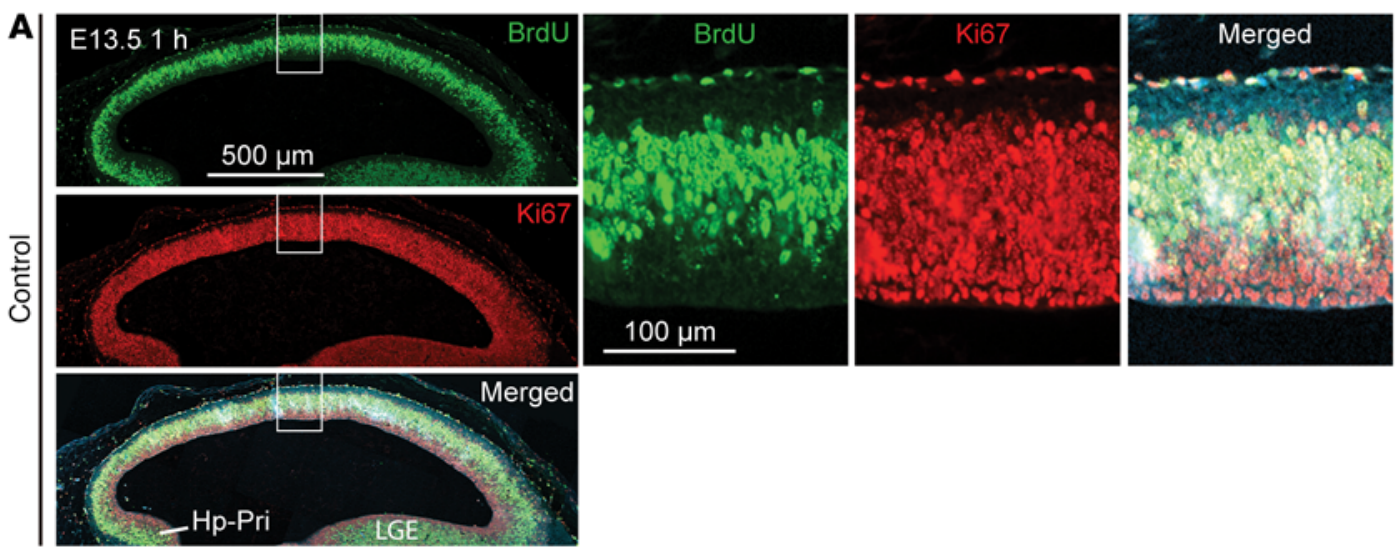

Figure 4. Cerebral Kat8 deletion impairs cell proliferation. (A) Immunostaining analysis of E13.5 control embryonic sections with anti-BrdU and -Ki-67 antibodies. Enlarged images of the boxed areas are shown at the middle and right. For BrdU labeling, timed mating was carried out, and BrdU was injected intraperitoneally into E13.5 pregnant mice. After 1 hour, the mice were euthanized for embryo retrieval, genotyping, section
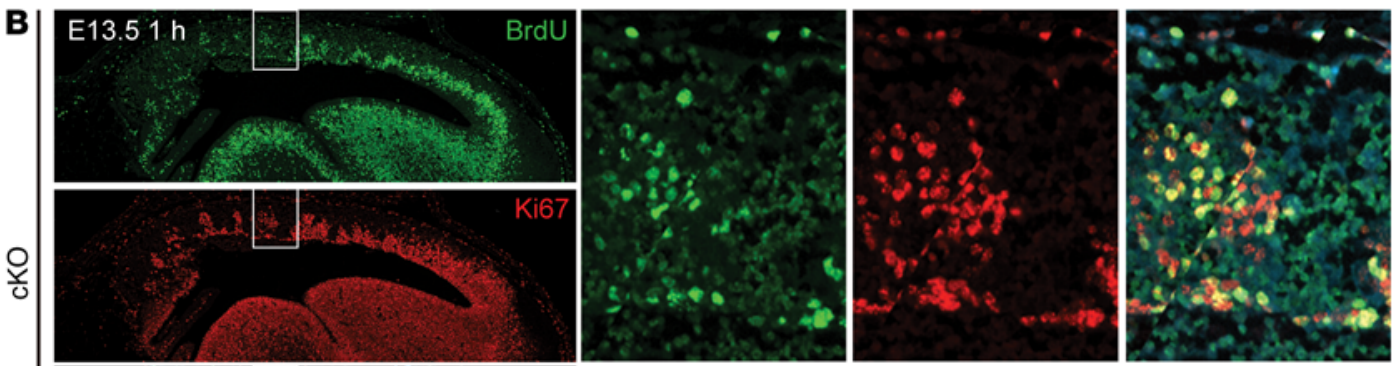
preparation, and subsequent immunostaining with the antibodies. (B) Same as $\mathbf{A}$ but mutant embryonic sections were analyzed. Images are representative of 3 experiments. Scale bars: 500

인

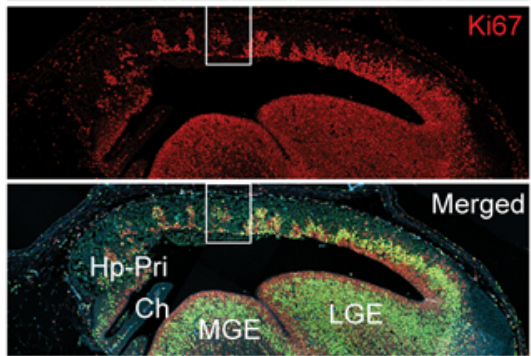
$\mu \mathrm{m}$ (left), $100 \mu \mathrm{m}$ (middle and right).

An alternative explanation is that the 2 variants from individual T9 and her family act differently from the de novo variants from individuals T1-T8. Related to this, dual-mode inheritance has been reported for some genes. For example, both monoallelic and biallelic EMC1 (and several other genes) variants cause developmental disorders (41).

Global developmental delay, intellectual disability, epilepsy, and other developmental anomalies were frequently observed in the affected individuals (Figure 7B, Supplemental Figure 8, and Supplemental Table 1), along with variable facial dysmorphism (Figure 7C and Supplemental Figure 8A). As for epilepsy, some exhibited EEG abnormalities (Supplemental Table 1). Variable language delay occurred in all individuals (Figure 7B and Supplemental Table 1): T5 had difficulty with pronunciation and could only form sentences at age 4; T6 did not speak and could only follow simple commands at age 2 ; T7 pronounced only 1 word at age 2; T8 only formed simple and short sentences at age 5; and T9 spoke no words at age 4 . Two individuals also showed autistic features (Figure 7B), and 1 individual displayed characteristics of attention deficit hyperactivity disorder (T2, Supplemental Table 1). Gross and fine motor delays were other common anomalies (Figure 7B and Supplemental Table 1). Four individuals had cardiac anomalies (Figure 7B and Supplemental Table 1). Cranial and facial abnormalities were also observed in some individuals. Recurrent dysmorphisms included upper lateral eyelid fullness, low-set ears, downturned corners of the mouth, a depressed nasal bridge, mild malar hypoplasia, and epicanthi (Figure 7B and Supplemental Table 1). Several individuals showed brain MRI anom- alies (Figure 7B; Supplemental Figure 8C; Supplemental Figure 9, A and B; and Supplemental Table 1). For the individuals with epilepsy, 2 of them have been treated with valproate and both were responsive (Figure 7B and Supplemental Table 1). Valproate is a known deacetylase inhibitor and promotes histone acetylation (42), so it may ameliorate potential acetylation deficiency resulting from KAT8 impairment (see below). Intellectual disability, epilepsy/seizures (Figure 7B and Supplemental Table 1), and variable brain MRI defects in the clinical cases (Supplemental Figure 8C and Supplemental Figure 9, A and B) suggest abnormal brain development, which is consistent with cerebral hypoplasia in the knockout mice (Figure 1).

Patient-derived variants inactivate KAT8 through different mechanisms. KAT8 is evolutionarily conserved from flies to humans (Supplemental Figure 10A). It is predicted to be moderately intolerant of missense change (misZ $[Z$ score of missense variation intolerance] score of 3.66) and highly intolerant of loss-of-function changes (pLI [probability of loss-of-function intolerance] score of 0.91, Exome Aggregation Consortium database). Among the 8 variants, 7 are missense (Figure 7A and Figure 8A). These missense variants alter key residues within the chromobarrel and enzymatic domains of KAT8 (Figure 8A). The residues are invariant in fly Mof, a KAT8 ortholog (Supplemental Figure 10A), suggesting their importance. According to a 3D structural model, they are all on the same plane (Figure 8B). The recurrent variant in individuals $\mathrm{T} 1, \mathrm{~T} 2$, and T3 alter Tyr90, whereas the missense variants in individuals T4 and T5 substitute Arg98 and Arg99, respectively. Tyr90, Arg98, and Arg99 are key residues of the chromobarrel domain (Figure 

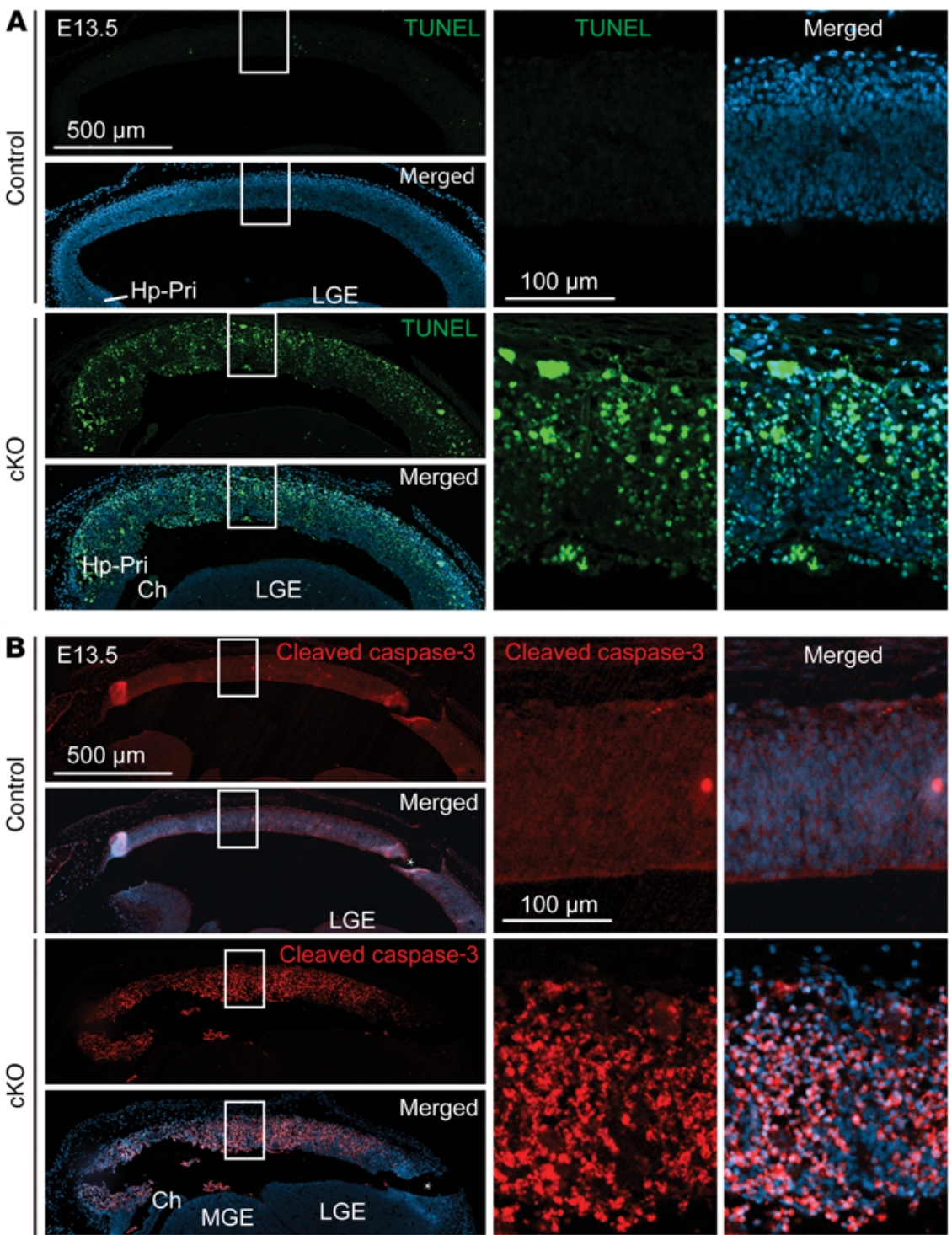

Figure 5. Cerebral Kat8 deletion triggers massive apoptosis. (A) TUNEL staining of E13.5 embryonic sections uncovered massive apoptosis at the mutant cerebrocortical neuroepithelia. (B) Immunostaining analysis of E13.5 embryonic sections with an anti-activated caspase-3 antibody confirmed massive apoptosis at the mutant cerebrocortical neuroepithelia. Images are representative of 2 experiments. Scale bars: $500 \mu \mathrm{m}$ (left), $100 \mu \mathrm{m}$ (middle and right).

8A and Supplemental Figure 10A). The function of this domain of KAT8 remains unclear, but the equivalent domain in fly Mof mediates nucleosome association (43). According to crystal structural analysis, this domain resembles chromodomains that recognize methylated histones (44). Moreover, a recent cryo-electron microscopy structural study has revealed that a similar domain of the yeast Esa1 acetyltransferase (another MYST family member) contributes to nucleosomal recognition and acetylation by its multisubunit complex (45). Tyr90, Arg98, and Arg99 of KAT8 are important for the structure of its chromobarrel domain (44), so their substitutions are likely deleterious. Structural modeling indicates that these residues are located at an interface with the catalytic domain (Figure 8B), so substitution of Tyr90, Arg98, or Arg99 may impede interaction with the catalytic domain and affect its acetyltransferase activity, especially toward nucleosomes. The missense variants in individuals T6-T8 alter Ala165, Lys175, and Lys181 within the catalytic domain (Figure 8A and Supplemental Figure 10A). These 3 residues are invariant in Drosophila Mof (Supplemental Figure 10A) and located within a region not conserved in the acetyltransferase domains of other MYST proteins, suggesting that this region may possess KAT8/Mof-specific functions. Notably, this region contains at least 5 lysine residues for acetylation (18), with 1 of them being Lys175 (Supplemental Figure 10A). Individual T9 carries 2 mutant alleles. One of them contains a missense variant that alters Arg325 (Figure 7A and Figure 8A). This residue is located within a known acetyl-CoA binding motif (Supplemental Figure 10A) and is structurally close to Arg98 and Arg99 (Figure $8 B$ ), so cysteine substitution may inactivate KAT8. The other allele encodes a nonsense variant (Figure 7A, c.523A $>\mathrm{T}$ ), generating $\mathrm{C}$-terminal truncation and removing the catalytic domain (Figure 8A and Supplemental Figure 10B, p.Lys $175^{*}$ ). This variant should be thus defective. Moreover, this but not the missense variant formed abnormal subnuclear localization (Figure 8C). Therefore, the 8 variants reported here form distinct groups (Figure 8A) and may deregulate KAT8 functions through different mechanisms.

To assess impact of the variants experimentally, we determined acetyltransferase activities of the variants. KAT8 forms 2 stoichiometric multisubunit complexes, 1 of which is tetrameric and contains malespecific lethal 1 (MSL1), MSL2, and MSL3 as noncatalytic subunits (Supplemental Figure 10B). Because MSL proteins directly activate the acetyltransferase activity of KAT8 toward H4K16 (46), we expressed wild-type KAT8 and its variants as FLAG-tagged fusion proteins along with HA-tagged MSL1, MSL2, and MSL3 for affinity purification on anti-FLAG M2 agarose. Purified complexes were then used for acetylation of recombinant nucleosomes and subsequent detection of histone $\mathrm{H} 4$ acetylation by immunoblotting. Although p.Lys $175^{*}$ was difficult to express (Figure $8 \mathrm{D}$, top panel, lane 13), the 7 missense variants were expressed to similar levels as wild-type KAT8 (Figure 8D, top panel, lanes 4-12). Like the wild-type, these missense variants promoted expression of MSL proteins in HEK293 cells (Figure 8D, second panel from the top, lanes 4-12). In contrast, p.Lys175* was unable to do so (lane 13). The missense variants also formed complexes with MSL proteins as wild-type KAT8 (panels 3-4 from the top, lanes 4-12). Unlike wild-type KAT8, the variants were defective in acetylating histone $\mathrm{H} 4$ at lysine 5 or 16 when recombinant nucleosomes were used as the substrate (bottom 3 panels), although the effects on lysine 5 acetylation were less dramatic for some 
A E16.5 embryonic neurospheres at day 4

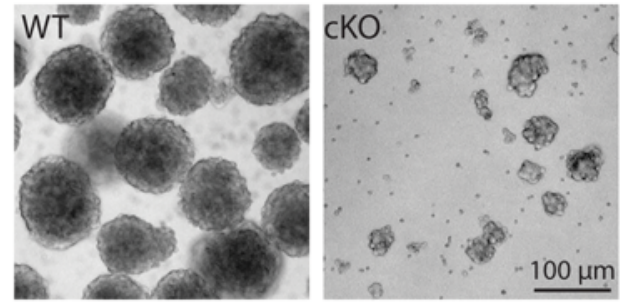

B Wild-type E18.5 neurospheres at day 6
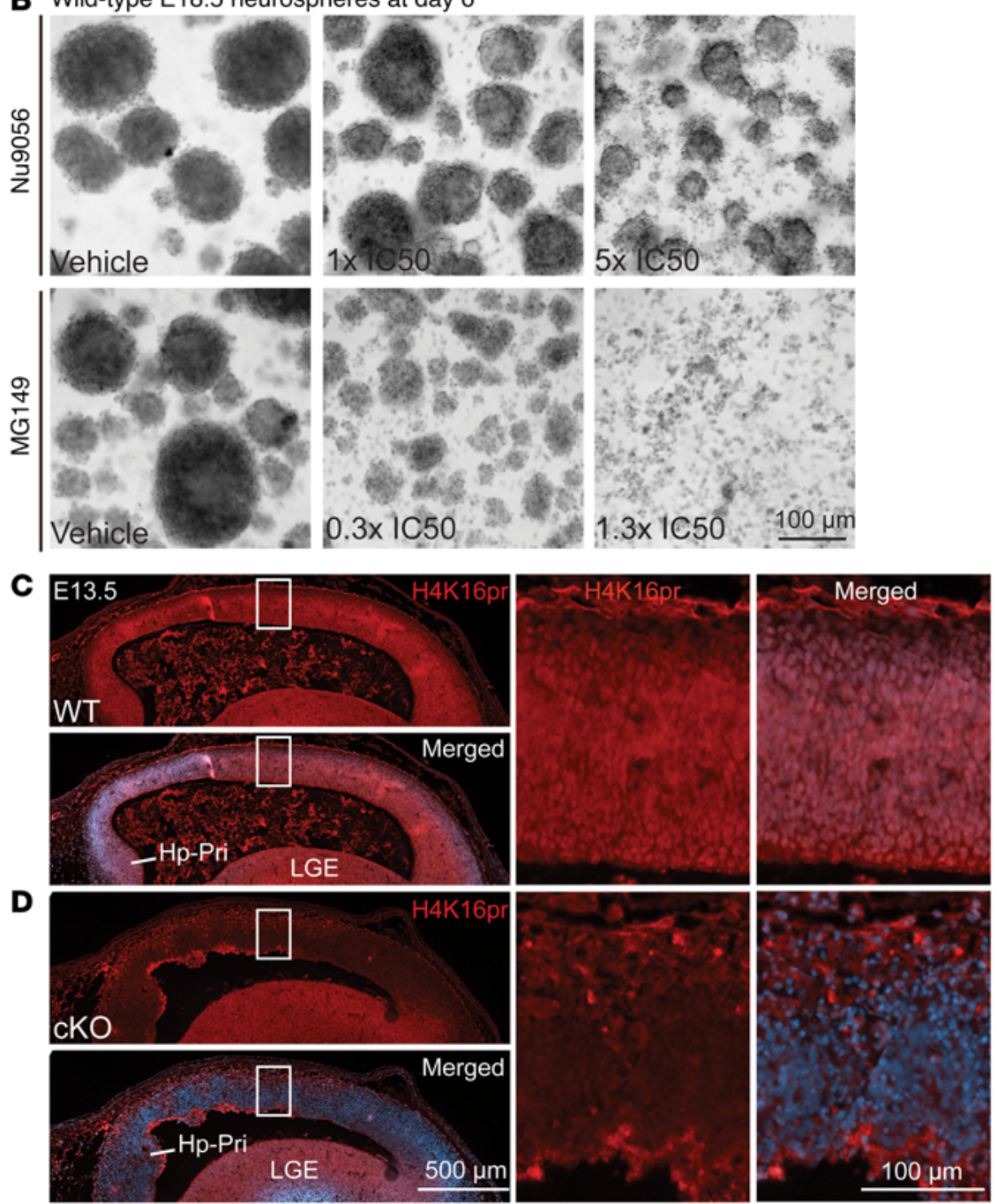

Figure 6. Critical roles of KAT8 in neurosphere formation and H4K16 propionylation. (A) Defective neurosphere formation from mutant but not wild-type NSPCs isolated from the cerebral cortex of the cerebrum-specific Kat8-knockout embryo at E16.5. (B) MG149 (targeting KAT5 and KAT8 with $\mathrm{IC}_{50}$ values of 74 and $47 \mu \mathrm{M}$, respectively) affected neurosphere formation more dramatically than NU9056 (targeting KAT5 at an IC $\mathrm{C}_{50}$ value of $2 \mu \mathrm{M}$ ). The inhibitors were added at the indicated concentrations (according to $\mathrm{IC}_{50}$ values against KAT5) after single cells were seeded for neurosphere formation. The vehicle (DMSO) amount was maintained at the same amount for all conditions. (C and $\mathbf{D}$ ) Immunostaining of E13.5 wild-type (C) and mutant (D) embryonic sections with the anti-H4K16pr antibody to detect H4K16 propionylation ( $\mathrm{pr}$ ). Results are representative of 3 (A and B) or 2 (C and $\mathbf{D})$ experiments.

variants. The severe impact of p.Tyr90Cys, p.Arg98Gln, and p.Arg99Gln on H4K16 acetylation (bottom 2 panels, lanes 5-8) suggests that the chromobarrel domain is essential for KAT8 to acetylate nucleosomal H4K16. To substantiate this, we analyzed an N-terminal truncation mutant lacking an intact chromobarrel domain (Supplemental Figure 10B, mutant 88-458). This mutant formed a similar complex with MSL proteins as wild-type KAT8 but did not acetylate nucleosomal H4K16 as efficiently as wildtype KAT8 did (Supplemental Figure 10C), further supporting importance of the chromobarrel domain in the acetylation activity. This is also consistent with the conclusion that the substitutions p.Tyr90Cys, p.Arg98Gln, and p.Arg99Gln impede the acetyltransferase activity of KAT8 (Figure 8D). Thus, the 7 missense variants (Figure 8A) are considered pathogenic because of defects in nucleosomal histone $\mathrm{H} 4 \mathrm{~K} 16$ acetylation.

\section{Discussion}

Results presented herein show that the mutant mice lacking Kat8 specifically in the cerebrum display early lethality before weaning at 3 weeks and show cerebral hypoplasia in the neocortex and hippocampus (Figure 1), at least in part due to poor development of NSPCs (Figure 3 and Supplemental Figure 4) and massive apoptosis (Figure 5). Mutant NSPCs failed to form neurospheres in vitro (Figure 6A), and KAT8 inhibition with a small-molecule inhibitor abolished neurosphere formation from wild-type NSPCs (Figure 6B). KAT8 is also essential for H4K16 propionylation in vivo (Figure 6, C and D and Supplemental Figure 7, C and D). In addition, human KAT8 deficiency, due mostly to de novo or occasionally to biallelically inherited variants, is associated with a new syndromic intellectual disability disorder (Figure 7, Figure 8, and Supplemental Table 1). Developmental delay, intellectual disability, epilepsy, and language impairment are consistent with cerebral hypoplasia in the mutant mice (Figure 1, Figure 2, Figure 3, Figure 4, and Figure $5)$. This study thus develops a mouse model, identifies human individuals with KAT8 variants, and uncovers the expected importance of the H4K16 acylation axis for mouse and human cerebral development (Figure 8E).

Multiple studies with cultured cells in vitro have established that KAT8 is critical for DNA damage responses $(9,15-18)$. By contrast, cerebrum-specific deletion exerted minimal effects on the $\gamma \mathrm{H} 2 \mathrm{~A} . \mathrm{X}^{+}$cell number (Supplemental Figure 5B). Related to this difference, we have not tested conditions where DNA damage is induced (e.g., by irradiation), so further analyses are needed to address this issue. Interestingly, Kat8 deletion triggered apoptosis at both E12.5 and E13.5 (Figure 5 and Supplemental Figure 6). In agreement with 
A

$\mathrm{T} 7(\mathrm{c} .523 \mathrm{~A}>\mathrm{G})$

T4 (c.293G>A) T6 (c.494C>T) T9 (c.523A>T) Nonsense

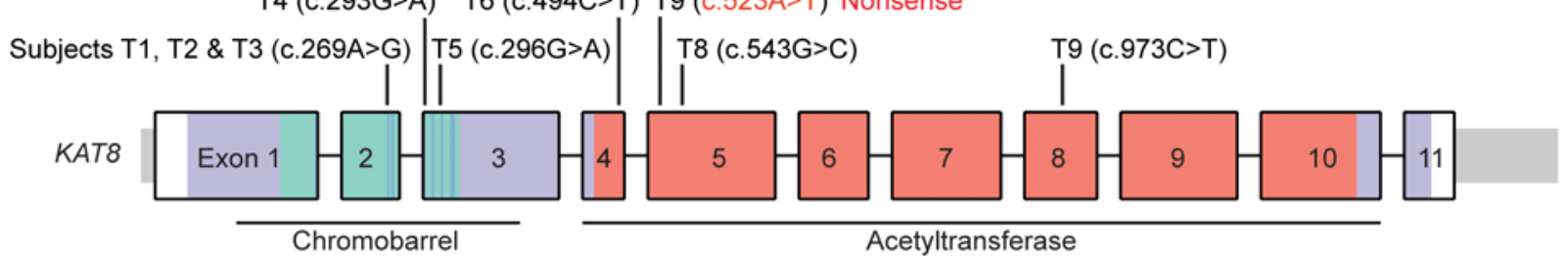

B
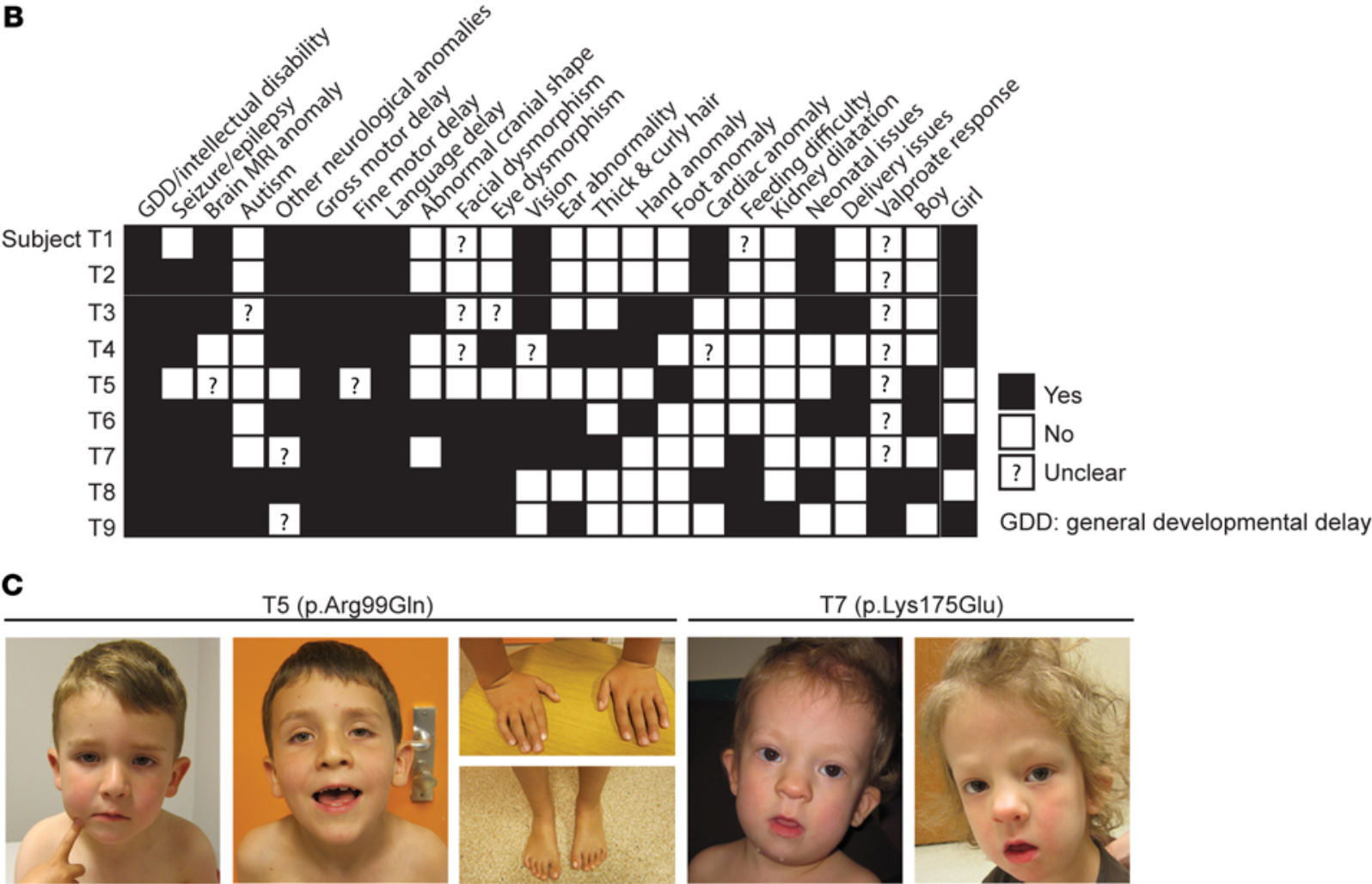

T5 (p.Arg99GIn)
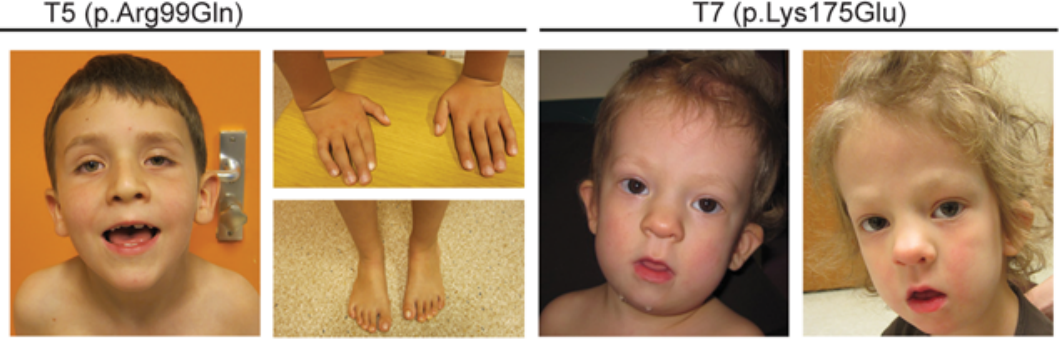

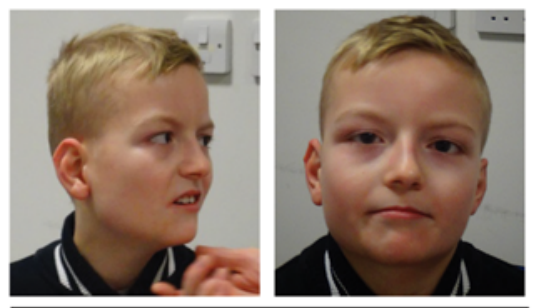

T8 (p.Lys181Asn)
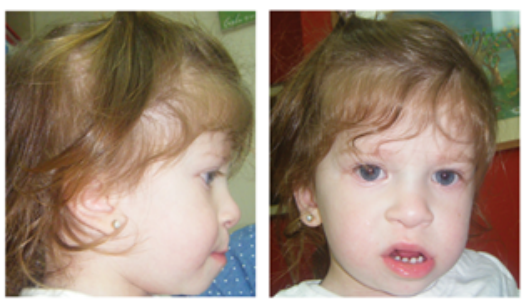

T9 (p.Lys175*/p.Arg325Cys)

Figure 7. KAT8 variants, clinical features, and photographs of 9 different individuals. (A) Exon-intron structure of KAT8 and the location of its variants identified in 9 individuals. All except individual T9 carry heterozygous de novo variants. Individuals T1, T2, and T3 are not related but share a recurrent variant. All except c.523A $>$ T are missense variants. c.523A $>$ T is a nonsense variant and converts codon 176 to a termination one. The regions encoding the chromobarrel and acetyltransferase domains of KAT8 are labeled with horizontal bars. (B) Heatmap comparing major clinical features in the 9 individuals. See Supplemental Table 1 for more detailed clinical information. (C) Photographs of 4 individuals. See Supplemental Figures 8 and 9 for photographs of individual T3 and MRI images of individuals T3, T6, T7, and T9.

this, inducible Kat8 deletion triggers apoptosis in myeloid cells (47). Cerebrum-specific Kat8 deletion affected NSPC proliferation (Figure 4) and neurogenesis at E13.5 (Figure 2A), but not so obvious E12.5 (Figure 2B and Supplemental Figure 5A), indicating context-dependent effects. Further studies are needed to gain mechanistic insights into molecular mechanisms whereby KAT8 regulates cellular programs important for cerebral development.
KAT8 is the catalytic subunit of 2 multiprotein complexes important for genome-wide epigenetic regulation (Supplemental Figure 10B) $(48,49)$, raising the intriguing question of how they contribute to the critical role of KAT8 in regulating cerebral development (Figure 8E). Subunits of the complexes are well expressed in the cerebral cortex of neonatal mice and neurospheres cultured from mouse embryos (Supplemental Figure 1, B and C) 
A

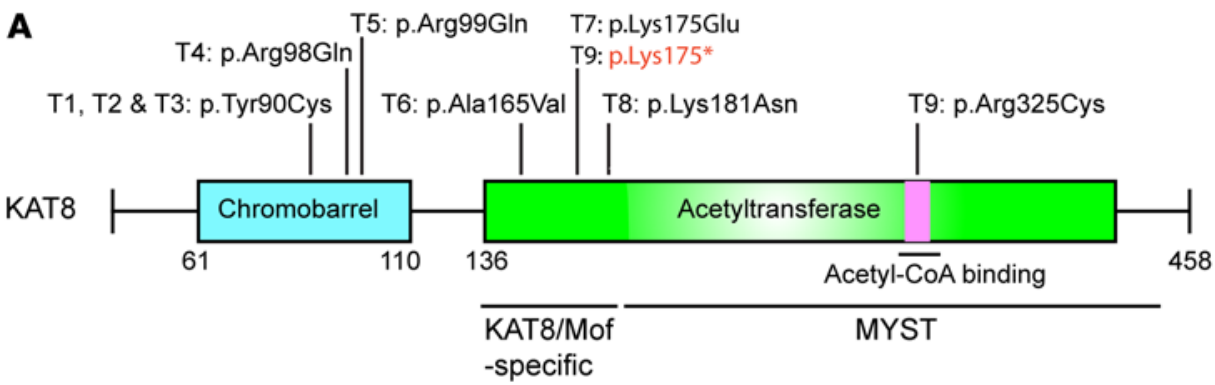

B
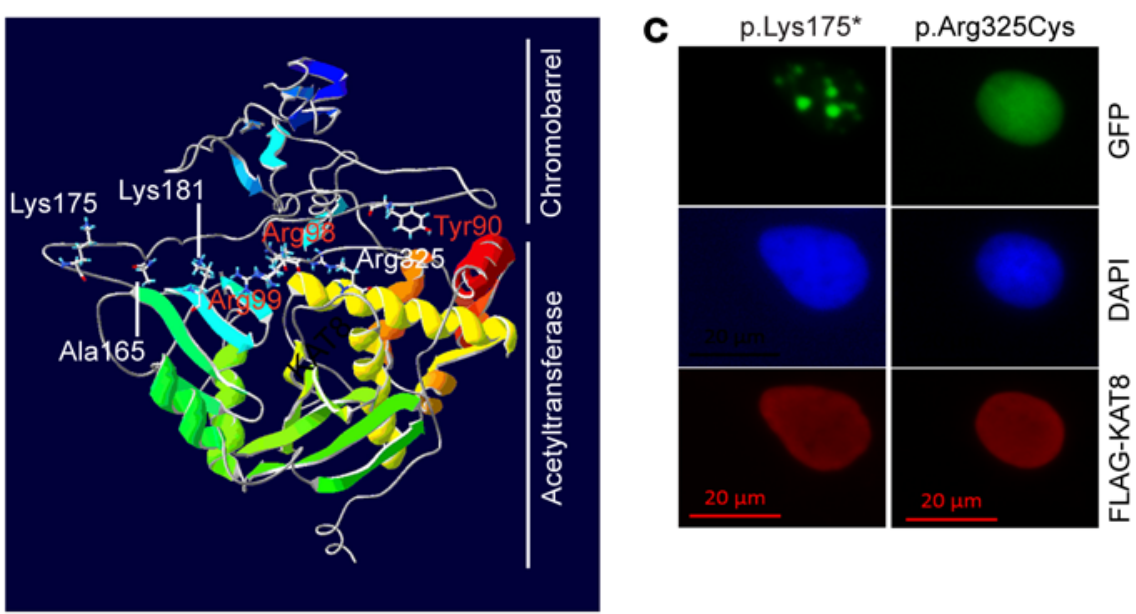

D

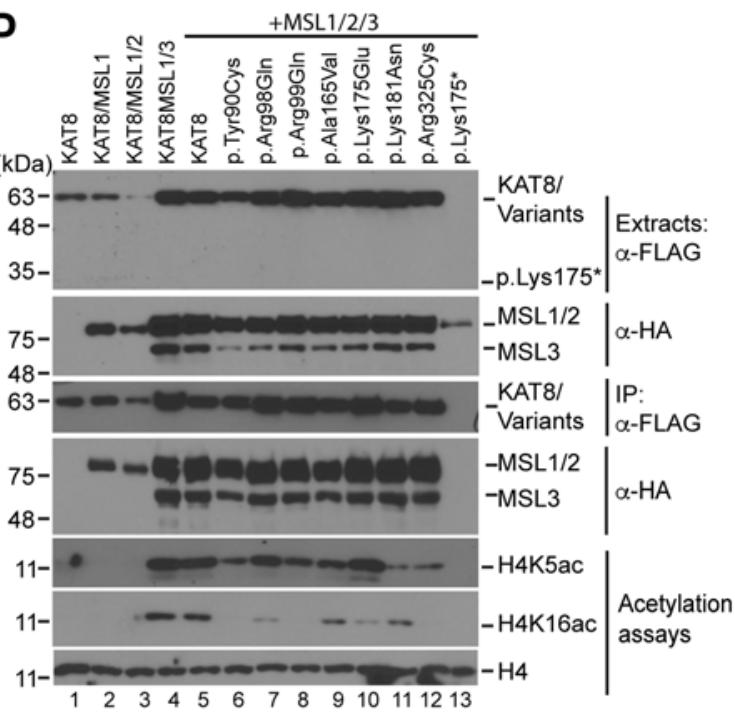

E

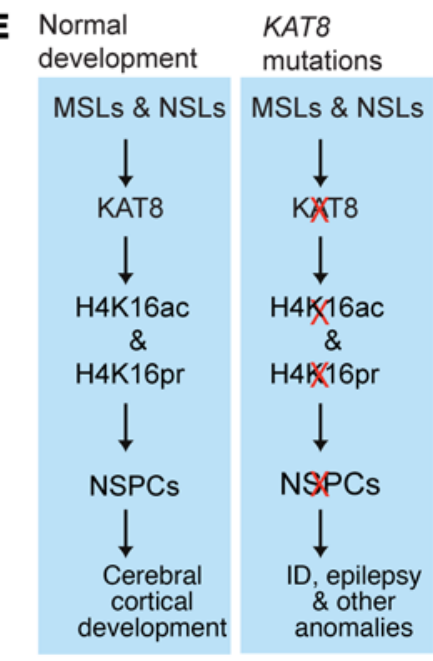

Figure 8. Characterization of 8 KAT8 variants associated with developmental anomalies. (A) Schematic representation of KAT8 and patient-derived variants (see Figure 7A and Supplemental Table 1 for the corresponding DNA sequence changes). KAT8 possesses a chromobarrel domain and an acetyltransferase core, both of which are conserved in fly Mof (Supplemental Figure 10A). The acetyltransferase domain is composed of a KAT8/Mof-specific region (absent in other members of the MYST family) and a MYST domain. The acetyl-CoA binding site is indicated. Among the missense variants, 3 alter the chromobarrel domain, and 4 change the acetyltransferase domain. Among the latter group, 3 affect residues located in the KAT8-specific region, and the fourth alters Arg325 within the acetyl-CoA binding motif. (B) Location of the affected residues on a 3D model generated from crystal structures of the chromobarrel and acetyltransferase domains of KAT8 or orthologs. (C) SubcelIular localization of the variants p.Lys $175^{*}$ and p.Arg325Cys. They were expressed as GFP-tagged proteins (shown in green) in HEK293 cells with FLAG-tagged wild-type KAT8 for immunostaining with anti-FLAG M2 antibody (shown in red). The subcellular localization of p.Lys175* but not p.Arg325Cys is different from the wild-type. DAPI stains nuclei (shown in blue). (D) Nucleosomal histone $\mathrm{H} 4$ acetylation assays showing that the missense variants are inactive in H4K16 acetylation. Impact on $\mathrm{H} 4 \mathrm{~K} 5$ acetylation was also affected, to a lesser extent. Extract preparation, complex purification and acetylation assays were carried out as in Supplemental Figure 10C. Results are representative of 2 (C) or 3 (D) experiments. (E) Schematic illustrating how KAT8 regulates cerebral and NSPC development through H4K16 acylation in normal individuals (left) and patients with KAT8 variants (right). In the patients, the regulatory axis is impaired, leading to intellectual disability, epilepsy, and other anomalies. MSL and non-specific lethal (NSL) proteins form stoichiometric complexes with KAT8 (Supplemental Figure 10B) and enhance its acyltransferase activities.
(50), suggesting involvement of both complexes in regulating cerebral development. In support of this, KANSL1 (encoding the key KAT8-associated subunit NSL1) is altered in multiple individuals with syndromic intellectual ability $(51,52)$. Even though the significance remains unclear, partial KANSL1 duplication is present in some Europeans (53). Moreover, a recent study identified MSL3 variants in individuals with an X-linked developmental disorder with intellectual disability (54). MSL3 cooperates with MSL1 and MSL2 in KAT8 activation (Figure 8D, lanes 1-5) (55). Notably, individuals with KAT8 variants (Figure 7 and Supplemental Table 1) variably exhibit epilepsy and other abnormalities absent in individuals with MSL3 variants (54). These studies suggest that KAT8 may act through both MSL and NSL complexes
(Supplemental Figure 10B) to control H4K16 acylation during cerebral development (Figure 8E).

The potential of KAT8 to catalyze $\mathrm{H} 4 \mathrm{~K} 16$ propionylation is intriguing (Figure 6, C and D, and Supplemental Figure 7, C and D) (36). The acetyl-CoA concentration is much higher than propionyl-CoA in vivo (56), so propionylation may function as a complementary mechanism under conditions when the propionyl-CoA level is elevated (Supplemental Figure 7E). The relative concentration of propionyl-CoA is also higher in the liver than heart, kidney, brain, and muscle (56), so the importance of propionylation may vary from tissue to tissue. Notably, subnuclear distribution of H4K16pr is different from H4K16ac (Supplemental Figure 7, A and B). Clinically, the propionyl-CoA level is elevated 
in cases of propionic acidemia, a rare recessive genetic disorder (57). In such cases, there may be hyperpropionylation (58). As for functional difference of H4K16 acetylation and propionylation, they may recruit protein readers differently, as has been shown for acetylation and crotonylation of histone $\mathrm{H} 3$. These interesting issues deserve further investigation.

Some other acetyltransferases have been linked to neurodevelopmental disorders. Pathogenic variants of CREBBP and EP3OO (encoding CBP and p300, respectively, required for histone H3 acetylation at lysine 27 in vivo) (59) cause Rubinstein-Taybi syndrome with learning difficulties (60). Moreover, KAT6A or KAT6B deficiency is linked to neurodevelopmental disorders with intellectual disability and global developmental delay (11). In addition, the multidomain scaffold BRPF1 activates KAT6A and KAT6B for acetylation of histone $\mathrm{H} 3$ at lysine 23 (61-63), and individuals with $B R P F 1$ variants exhibit syndromic intellectual disability $(5,64)$. Among the remaining 2 MYST family members, KAT5 is responsible for acetylation of histones $\mathrm{H} 4$ and H2A.Z, whereas KAT7 is the major acetyltransferase acetylating histone $\mathrm{H} 3$ at lysine 14 (3). It remains unclear how KAT5 and KAT7 may regulate cerebral development or whether their variants are linked to any developmental disorders. Answers to these 2 questions will help understand how different acetyltransferases confer epigenomic dynamics and plasticity during cerebral development.

This study is about KAT8 as an essential H4K16 acetyltransferase for cerebral development, so a related question is what deacetylases are involved in H4K16 deacetylation. Early studies established that in yeast, Sir2 antagonizes Sas2 (a KAT8 ortho$\log$ ) and controls H4K16 acetylation and heterochromatin spread (65), suggesting that a similar mechanism may operate in higher organisms. Indeed, some evidence supports that mammalian Sir2related proteins, such as SIRT1, serve as H4K16 deacetylases $(66,67)$. For example, inducible Sirt1 deletion enhances H4K16 acetylation in hematopoietic cells and (68) and some Sirt1-null embryos show cerebral defects (69). Moreover, Sirt2 is highly expressed in the neonatal cerebral cortex and E16.5 embryoderived neurospheres (50). There is also evidence supporting the involvement of SIRT2 as an H4K16 deacetylase at least in cultured cells (70). However, treatment with classical deacetylase inhibitors enhances H4K16 acetylation $(54,71)$, suggesting the involvement of additional deacetylases. Related to this, histone deacetylase 1 (HDAC1), HDAC2, and HDAC3 are all important for cerebral development $(50,72,73)$. Among them, Hdac2 is very highly expressed in the neonatal cerebral cortex and embryoderived neurospheres (50), suggesting a potentially prominent role of this deacetylase in cerebral development. Notably, even cerebrum-specific deletion of Hdac3 leads to H4K16 hyperacetylation (50). Thus, several deacetylases may control H4K16 acetylation during cerebral development. In addition, KAT8 is activated by autoacetylation $(74,75)$, while SIRT1 is known to deacetylate KAT8 and inhibit its activity $(18,76)$. These issues are clinically relevant, because in addition to association with the developmental disorder described herein (Figure 7 and Figure 8), H4K16 acetylation is altered during aging and in Alzheimer's disease (77).

In summary, we have identified an important role of mouse Kat 8 in cerebral development and provided evidence that 8 KAT 8 variants are associated with intellectual disability, epilepsy, and other anomalies. This report highlights the importance of KAT8 and its complexes (Supplemental Figure 10B) in human development and sheds light on functions of their orthologs in different organisms. The findings also suggest the potential value of using Kat8-mutant mice (Figure 1) as preclinical models for exploring therapeutic options, e.g., for testing deacetylase inhibitor drugs such as valproic acid (Figure 7B). This study thus uncovers an intimate link of syndromic intellectual disability disorders to deficient histone acylation and reiterates the importance of epigenetic regulation for cerebral and intellectual development in humans.

\section{Methods}

Exome and Sanger sequencing. Exome sequencing was carried out as described previously (5). Specifically, whole-exome sequencing of individual $\mathrm{T} 5$ was performed with the SeqCap EZ MedExome kit (Roche), and sequencing was performed on a HiSeq 2500 (Illumina); an in-house bioinformatic pipeline was used to identify variants. For individual T8 and the parents, trio-based whole-exome sequencing was undertaken through the Deciphering Developmental Disorders (DDD) study (78). After exome sequencing, PCR fragments were amplified from genomic DNA or reverse-transcribed cDNA to confirm KAT8 variants. For exome sequencing at GeneDx, genomic DNA from the proband and parents were used to capture the exonic regions and flanking splice junctions of the human genome via the Clinical Research Exome kit (Agilent Technologies) or the Integrated DNA Technologies xGen Exome Research Panel v1.0. Massively parallel (next-generation) sequencing was carried out on an Illumina system with 100-bp or greater paired-end reads. Reads were aligned to human genome build GRCh37/UCSC hg19 and analyzed for sequence variants using a custom-developed analysis tool. The general assertion criteria for variant classification are available on the GeneDx ClinVar page (http://www.ncbi.nlm.nih.gov/clinvar/submitters/26957/).

Lymphoblastoid cell preparation. Lymphoblastoid cells were established and cultured as described previously (5).

Mice. The Kat8 $8^{f l}$ allele contains 2 LoxP sites within the Kat8 gene, as has been described (21). Cerebrum-specific knockouts were generated by mating $\mathrm{Kat}^{\mathrm{fl} / \mathrm{fl}}$ mice with the Emx1-Cre strain (The Jackson Laboratory, 005648), as previously described for the Brpf1 gene (28, 79-81).

Immunoprecipitation and histone $\mathrm{H} 4$ acetylation assays. An expression plasmid for FLAG-tagged KAT8 was transfected into HEK293 cells (ATCC, CRL-1573) along with expression vectors for HA-tagged MSL proteins as specified. About 48 hours after transfection, cells were washed twice with PBS, and soluble protein extracts were prepared for affinity purification on the anti-FLAG M2 agarose (MilliporeSigma, A2220) as described $(62,82)$. The FLAG peptide (Millipore Sigma, F3290) was used to elute bound proteins for immunoblotting with anti-FLAG and -HA antibodies or for acetyltransferase activity determination. Acetylation of recombinant nucleosomes (EpiCypher, SKU16-0009) or histone octamer (EpiCypher, SKU 16-0001) was performed as described $(62,82)$. After acetylation reactions, immunoblotting analyses were carried out, as described below, to detect histone $\mathrm{H} 4$ or its site-specific acetylation by use of anti-histone H4 (Abcam, ab18253), -H4K5ac (MilliporeSigma, 07-327), -H4K8ac (MilliporeSigma, 07-328), -H4K12ac (MilliporeSigma, 07-595), and -H4K16ac (MilliporeSigma, 07-329) antibodies. The SuperSignal West Pico PLUS Chemiluminescent Substrate (Pierce, Thermo Fisher 
Scientific, 34580) or the SuperSignal West Femto Maximum Sensitivity Substrate (Pierce, Thermo Fisher Scientific, 34095) were used to develop the signals.

Immunoblotting. On ice, neurospheres were suspended in cold RIPA buffer (150 mM NaCl, 1.0\% Nonidet P-40 or Triton X-100, $0.5 \%$ sodium deoxycholate, $0.1 \%$ SDS, $50 \mathrm{mM}$ Tris- $\mathrm{HCl}, \mathrm{pH}$ 8.0) containing a proteinase inhibitor cocktail, composed of $1 \mathrm{mM}$ phenylmethylsulfonyl fluoride, $1 \mu \mathrm{g} / \mathrm{mL}$ pepstatin, $2 \mu \mathrm{g} / \mathrm{mL}$ aprotinin, and $0.5 \mu \mathrm{g} /$ $\mathrm{mL}$ leupeptin. After brief sonication (for 15 seconds at setting 5 with a VirSonic 100 Ultrasonic Cell Disrupter, SP Scientific) on ice, the suspension was centrifugated in an Eppendorf centrifuge for 10 minutes at $20,000 \mathrm{~g}$ and $4^{\circ} \mathrm{C}$. The supernatant was transferred to a new tube on ice. The protein concentration was determined by using a Bradford protein assay kit (MilliporeSigma). Protein extracts were mixed with a $3 \times$ reducing sample buffer $(240 \mathrm{mM}$ Tris- $\mathrm{HCl}$ at $\mathrm{pH} 6.8,6 \%$ SDS, $30 \%$ glycerol, $16 \% \beta$-mercaptoethanol, $0.06 \%$ bromophenol blue) and boiled for 8 minutes, for SDS-PAGE on $10 \%-15 \%$ acrylamide gels and subsequent transfer onto a nitrocellulose membrane (Pall Corporation, $\mathrm{P} / \mathrm{N} 66485)$. The membrane was incubated with the blocking buffer TBS supplemented with $0.1 \%$ Tween-20 (TBS-T) containing $5 \%$ nonfat milk powder for 30 minutes at room temperature on a rocking platform and then incubated with the same buffer containing primary antibodies overnight at $4^{\circ} \mathrm{C}$ on a rocking platform. After washing with TBS-T 4 times (10 minutes each, with agitation), the membrane was incubated, on a rocking platform, with the blocking buffer containing the horseradish peroxidase-conjugated secondary antibodies (GE Healthcare Life Sciences, NA931V and NA934V) at room temperature for 1 hour. Alternatively, TBS-T was replaced with PBS containing $0.15 \%$ Tween-20. The membrane was washed as above and developed with the enhanced chemiluminescence substrates (FroggaBio, 16024), the SuperSignal West Pico PLUS Chemiluminescent Substrate (Pierce, Thermo Fisher Scientific, 34580), or the SuperSignal West Femto Maximum Sensitivity Substrate (Pierce, Thermo Fisher Scientific, 34095).

Green and indirect fluorescence microscopy. For analysis of subcellular localization, expression plasmids for EGFP-tagged KAT8 variants were transiently transfected into HEK293 cells with or without plasmids for FLAG-tagged wild-type KAT8 as specified. About 16 hours after transfection, cells were fixed for indirect fluorescence microscopy with the anti-FLAG M2 antibody (MilliporeSigma, F3165). Fluorescence signals were examined under a fluorescence microscope (Axio Observer Z1, Zeiss), and fluorescence images were captured for further processing as described (82).

Histology. Mouse brain and embryos were dissected out and fixed in $4 \%$ paraformaldehyde (PFA) for 24-48 hours, as previously described $(28,79,83)$. The tissues were then subjected to dehydration and paraffin embedding. The paraffin blocks were sectioned to $5 \mu \mathrm{m}$ and used for subsequent Nissl staining, H\&E staining, or immunofluorescence microscopy as described $(28,79,83)$. The antibodies used for immunostaining were goat anti-SOX2 (R\&D Systems, AF2018, 1:200), rabbit anti-TBR2 (Abcam, ab23345, 1:400), rat anti-CTIP2 (Abcam, ab18465, 1:400), rabbit anti-CUX1 (provided by Alain Nepveu, McGill University, 1:200) (84), mouse anti-Tuj1 (Covance, MMS-435p, 1:1000), rat anti-BrdU (Abcam, ab6326, 1:400), mouse anti-Ki-67 (BD Pharmingen, 550609, 1:200), rabbit anti-H4K16ac (Abcam, ab109463, 1:1500), mouse anti-H4K16pr (PTM Biolabs, PTM-210, 1:1000), and rabbit anti- $\gamma$ H2A.X (Abcam, ab2893, 1:500) antibodies.
BrdU tracing in vivo. BrdU labeling was carried out as described $(28,79)$. BrdU was injected intraperitoneally into pregnant mice at a dosage of $50 \mathrm{mg}$ per $\mathrm{kg}$ of body weight. The mice were euthanized at different time points according to experimental goals. For S-phase analysis, mice were euthanized 1 hour after injection.

Apoptosis detection. TUNEL assays were carried out with paraffin sections by use of the DeadEnd fluorometric TUNEL kit (Promega, G3250) according to the manufacturer's instructions. After deparaffinization and rehydration, embryonic sections were refixed with 4\% PFA for 10 minutes and washed once with PBS. The sections on slides were circled with a hydrophobic PAP pen to build a hydrophobic barrier for permeabilization with $50 \mu \mathrm{L}$ PBS (containing 0.2\% Triton $\mathrm{X}-100$ and $20 \mu \mathrm{g} / \mathrm{mL}$ proteinase $\mathrm{K}$ ) for 10 minutes at room temperature. The sections were then refixed in 4\% PFA again for 10 minutes, washed with PBS, and equilibrated with the equilibration buffer provided with the kit. After equilibration, the sections were incubated with $50 \mu \mathrm{L}$ of a terminal deoxynucleotidyl transferase reaction mix for 1 hour at $37^{\circ} \mathrm{C}$, and the reaction was stopped by immersion of the slides in $100 \mathrm{~mL}$ of $2 \times \mathrm{SSC}$ for 15 minutes at room temperature. The sections were then counterstained with $0.1 \mu \mathrm{g} / \mathrm{mL}$ DAPI (MilliporeSigma, D9542) in PBS for 15 minutes, and the slides were mounted with coverslips for further analysis under a fluorescence microscope.

To detect activated cleaved caspase-3, after deparaffinization, rehydration, and antigen retrieval (by boiling in a $10 \mathrm{mM}$ sodium citrate buffer at $\mathrm{pH}$ 6.0, containing 0.05\% Tween-20, for 20 minutes), the sections were incubated in a blocking buffer (PBS containing 2\% BSA and $0.2 \%$ Triton $\mathrm{X}-100$ ) at room temperature for 30 minutes, followed by incubation with rabbit anti-mouse cleaved caspase- 3 antibody (Cell Signaling Technology, 9661, 1:200) overnight at $4^{\circ} \mathrm{C}$ and an Alexa Fluor 568-conjugated secondary (Invitrogen, Thermo Fisher Scientific, A-11011, 1:500) for 1 hour at room temperature. Nuclei were counterstained with DAPI for slide mounting and subsequent examination under a fluorescence microscope.

Neurosphere formation assays. All the procedures were done in a tissue culture hood unless indicated otherwise. All the surgical instruments and solutions used were either autoclaved or filter sterilized. Briefly, pregnant mice at E16.5 were euthanized, and embryos were dissected out and transferred to PBS. The embryo head was collected, washed with PBS, and transferred to PBS/2\% glucose on ice for subsequent manipulation. Under a dissecting microscope, the head skin was peeled off, and the skull was opened with a pair of surgical scissors. The brain was dissected out with a pair of forceps. The cerebral cortices were segregated from the rest of the brain tissues and collected into Eppendorf tubes containing $1 \mathrm{~mL}$ of the complete neurosphere medium on ice. The medium contained mouse NeuroCult NSC Basal Medium (STEMCELL Technologies, 05700) supplemented with $10 \%$ NeuroCult Proliferation Supplement (STEMCELL Technologies, 05701) and $20 \mathrm{ng} / \mathrm{mL}$ human recombinant EGF (STEMCELL Technologies, 78006.1). Once all cortices were collected, the tubes were transferred into the cell culture hood and gently pipetted up and down with 1-mL Pipetman tips for 8 times to prepare single-cell suspensions (with the maximum pipetting volume set to $0.8 \mathrm{~mL}$ ). Air bubbles were avoided by pipetting gently because excessive oxygen from the bubbles would have been deleterious for NSPC growth. Afterward, the tubes were kept still at room temperature for 1 minute, and the supernatants were then transferred to new tubes for centrifugation at $150 \mathrm{~g}$ for 5 minutes with a bench centrifuge at room temperature. The super- 
natants were discarded, and each pellet was gently mixed with $1 \mathrm{~mL}$ of the fresh neurosphere medium. The suspension was filtered through a 40- $\mu \mathrm{m}$ nylon cell strainer (Thermo Fisher Scientific, 22363547), and the filtrate was considered a single-cell preparation. After counting, wild-type and mutant cells were seeded into wells of a 6-well plate at the same number. For treatment with the KAT5 and KAT8 inhibitors, NU9056 (Tocris Bioscience, $4903 ; 1 \times \mathrm{IC}_{50}[2 \mu \mathrm{M}]$ or $5 \times \mathrm{IC}_{50}[10 \mu \mathrm{M}]$, according to the $\mathrm{IC}_{50}$ value in TIP60 inhibition) and MG149 (Selleckchem, S7476; 0.3× $\mathrm{IC}_{50}[23.6 \mu \mathrm{M}]$ or $1.3 \times \mathrm{IC}_{50}[94 \mu \mathrm{M}]$, according to the $\mathrm{IC}_{50}$ value in TIP60 inhibition) were used. For immunoblotting, neurospheres were washed once with PBS for extract preparation in the RIPA buffer as described above.

Statistics. Statistical analysis was performed with unpaired 2-tailed Student's $t$ test. $P<0.05$ was considered statistically significant. Data represent mean \pm SEM. Graphs were generated with GraphPad Prism 8 (GraphPad Software).

Study approval. The clinical study was carried out according to a study protocol (protocol 4181) approved by CHU Sainte-Justine Institutional Review Board \#1 (Montreal, Quebec, Canada). The IRB at Boston Children's Hospital approved the family enrollment and human cell work on proband 6 (T6) under protocol 10-02-0253. The nonidentifying information of proband 1 (T1) was collected at an institute that did not require consent for publication of such information as an isolated case study. For the other 8 human subjects who participated in this clinical research study, written informed consent was received from the participants or their legal guardians. In addition, written informed consent was specifically obtained for the photographs presented in this manuscript. All biological samples from individual T8 and her family members were collected after obtaining written informed consent of the family, in agreement with the regulations of the IRBs (Beilinson Medical Center, Petach Tikva, Israel, and Hadasah Ein Karem, Jerusalem, Israel) and the Helsinki guidelines. Mouse-related procedures in this study were performed according to Animal Use Protocol 5786, which was reviewed and approved by the Facility Animal Care Committee of McGill University, Montreal, Quebec, Canada.

\section{Author contributions}

LL characterized the knockout mice, performed data analysis, and drafted the manuscript; MG analyzed patient-derived variants and discovered the mouse phenotype; MWH identified the proband family and initiated clinical studies; JR generated patient-derived cells; IT, RES, CB, KG, SW, HK, JAH, EMJB, LBS, OK, HGS, LB, ST, MMMW, QW, MS, GSB, SSB, SSH, RO, JMVH, and PBA identified individuals with $K A T 8$ variants and obtained clinical data; JJ repeated some assays at the manuscript revision stage; $\mathrm{XB}$ and $\mathrm{MB}$ helped with acetylation assays; TKP supplied the floxed mice; PMC initiated and supervised clinical studies; XJY generated knockout mice and coordinated the project; and with contributions from LL, MG, MWH, JR, IT, RES, CB, RO, MMMW, QW, SW, HK, JAH, EMJB, LBS, OK, HGS, LB, ST, JJ, XB, MB, KM, MTC, MS, GBS, SSB, SSH, KLIVG, JMVH, TKP, and PBA, PMC and XJY wrote and finalized the manuscript. LL, MG, and MWH made equally important contributions from 3 different angles of this project and are thus considered co-first authors.

\section{Acknowledgments}

This work was supported by research grants from the Natural Sciences and Engineering Research Council of Canada (to XJY), Canadian Institutes of Health Research (CIHR, to XJY and PMC), Cancer Research Society (to XJY), and National Institutes of Health (CA129537 and GM109768, to TKP). This study makes use of DECIPHER (http://decipher.sanger.ac.uk), which is funded by Wellcome Trust. The DDD study presents independent research commissioned by the Health Innovation Challenge Fund (grant number HICF-1009-003); see ref. 78 or www.ddduk.org/access. html for full acknowledgment. LL received stipend support from China Scholarship Council, the Clifford C.F. Wong Fellowship program, a CIHR/FRSQ training grant in cancer research for the McGill Integrated Cancer Research Training Program, and the Canderel Foundation.

Address correspondence to: Philippe M. Campeau, Medical Genetics Service, Room 4734, CHU Sainte-Justine Hospital, 3175 Côte-Sainte-Catherine, Montreal, Quebec H3T 1C5, Canada. Phone: 514.345.4931 ext.7146; Email: p.campeau@umontreal.ca. Or to: Xiang-Jiao Yang, Goodman Cancer Research Center, Room 413, 1160 Pine Avenue West, Montreal, Quebec H3A 1A3, Canada. Phone: 514.398.5883; Email: xiang-jiao.yang@mcgill.ca.
1. Allis CD, et al. New nomenclature for chromatinmodifying enzymes. Cell. 2007;131(4):633-636.

2. Dutta A, Abmayr SM, Workman JL. Diverse activities of histone acylations connect metabolism to chromatin function. Mol Cell. 2016;63(4):547-552.

3. Kueh AJ, Dixon MP, Voss AK, Thomas T. HBO1 is required for $\mathrm{H} 3 \mathrm{~K} 14$ acetylation and normal transcriptional activity during embryonic development. Mol Cell Biol. 2011;31(4):845-860.

4. Huang F, et al. Histone acetyltransferase Enok regulates oocyte polarization by promoting expression of the actin nucleation factor spire. Genes Dev. 2014;28(24):2750-2763.

5 . Yan $\mathrm{K}$, et al. Mutations in the chromatin regulator gene BRPF1 cause syndromic intellectual disability and deficient histone acetylation. Am J Hum Genet. 2017;100(1):91-104.

6. Shahbazian MD, Grunstein M. Functions of site-specific histone acetylation and deacetyla- tion. Annu Rev Biochem. 2007;76:75-100.

7. Gupta A, et al. The mammalian ortholog of Drosophila MOF that acetylates histone H4 lysine 16 is essential for embryogenesis and oncogenesis. Mol Cell Biol. 2008;28(1):397-409.

8. Kapoor-Vazirani P, Kagey JD, Powell DR, Vertino PM. Role of hMOF-dependent histone H4 lysine 16 acetylation in the maintenance of TMS1/ASC gene activity. Cancer Res. 2008;68(16):6810-6821.

9. Li X, et al. MOF and H4 K16 acetylation play important roles in DNA damage repair by modulating recruitment of DNA damage repair protein Mdc1. Mol Cell Biol. 2010;30(22):5335-5347.

10. Rea S, Xouri G, Akhtar A. Males absent on the first (MOF): from flies to humans. Oncogene. 2007;26(37):5385-5394.

11. Yang XJ. MOZ and MORF acetyltransferases: molecular interaction, animal development and human disease. Biochim Biophys Acta.
2015;1853(8):1818-1826.

12. Hilfiker A, Hilfiker-Kleiner D, Pannuti A, Lucchesi JC. mof, a putative acetyl transferase gene related to the Tip60 and MOZ human genes and to the SAS genes of yeast, is required for dosage compensation in Drosophila. EMBO J. 1997;16(8):2054-2060.

13. Gelbart ME, Kuroda MI. Drosophila dosage compensation: a complex voyage to the $\mathrm{X}$ chromosome. Development. 2009;136(9):1399-1410.

14. Kind J, et al. Genome-wide analysis reveals MOF as a key regulator of dosage compensation and gene expression in Drosophila. Cell. 2008;133(5):813-828.

15. Taipale M, et al. hMOF histone acetyltransferase is required for histone $\mathrm{H} 4$ lysine 16 acetylation in mammalian cells. Mol Cell Biol. 2005;25(15):6798-6810.

16. Thomas T, Dixon MP, Kueh AJ, Voss AK. Mof 
(MYST1 or KAT8) is essential for progression of embryonic development past the blastocyst stage and required for normal chromatin architecture. Mol Cell Biol. 2008;28(16):5093-5105.

17. Sharma GG, et al. MOF and histone H4 acetylation at lysine 16 are critical for DNA damage response and double-strand break repair. $\mathrm{Mol}$ Cell Biol. 2010;30(14):3582-3595.

18. Peng L, et al. SIRT1 negatively regulates the activities, functions, and protein levels of $\mathrm{hMOF}$ and TIP60. Mol Cell Biol. 2012;32(14):2823-2836.

19. Sykes SM, et al. Acetylation of the p53 DNA-binding domain regulates apoptosis induction. $\mathrm{Mol}$ Cell. 2006;24(6):841-851.

20. Yin S, et al. Histone acetyltransferase KAT8 is essential for mouse oocyte development by regulating reactive oxygen species levels. Development. 2017;144(12):2165-2174.

21. Kumar R, et al. Purkinje cell-specific males absent on the first (mMof) gene deletion results in an ataxia-telangiectasia-like neurological phenotype and backward walking in mice. Proc Natl Acad Sci U S A. 2011;108(9):3636-3641.

22. Chatterjee A, et al. MOF acetyl transferase regulates transcription and respiration in mitochondria. Cell. 2016;167(3):722-738.e23.

23. Gupta A, et al. T-cell-specific deletion of Mof blocks their differentiation and results in genomic instability in mice. Mutagenesis. 2013;28(3):263-270.

24. Valerio DG, Xu H, Eisold ME, Woolthuis CM, Pandita TK, Armstrong SA. Histone acetyltransferase activity of MOF is required for adult but not early fetal hematopoiesis in mice. Blood. 2017;129(1):48-59.

25. Huai W, et al. KAT8 selectively inhibits antiviral immunity by acetylating IRF3. JExp Med. 2019;216(4):772-785.

26. Gorski JA, Talley T, Qiu M, Puelles L, Rubenstein JL, Jones KR. Cortical excitatory neurons and glia, but not GABAergic neurons, are produced in the Emx1-expressing lineage. J Neurosci. 2002;22(15):6309-6314.

27. Chou SJ, Perez-Garcia CG, Kroll TT, O’Leary DD. Lhx2 specifies regional fate in Emx1 lineage of telencephalic progenitors generating cerebral cortex. Nat Neurosci. 2009;12(11):1381-1389.

28. You L, et al. Deficiency of the chromatin regulator BRPF1 causes abnormal brain development. J Biol Chem. 2015;290(11):7114-7129.

29. Noctor SC, Flint AC, Weissman TA, Dammerman RS, Kriegstein AR. Neurons derived from radial glial cells establish radial units in neocortex. Nature. 2001;409(6821):714-720.

30. Ellis P, et al. SOX2, a persistent marker for multipotential neural stem cells derived from embryonic stem cells, the embryo or the adult. Dev Neurosci. 2004;26(2-4):148-165.

31. Hodge RD, et al. Tbr2 is essential for hippocampal lineage progression from neural stem cells to intermediate progenitors and neurons. J Neurosci. 2012;32(18):6275-6287.

32. Arnold SJ, et al. The T-box transcription factor Eomes/Tbr2 regulates neurogenesis in the cortical subventricular zone. Genes Dev. 2008;22(18):2479-2484.

33. Singec I, et al. Defining the actual sensitivity and specificity of the neurosphere assay in stem cell biology. Nat Methods. 2006;3(10):801-806.

34. Ghizzoni M, Wu J, Gao T, Haisma HJ, Dekker FJ, George Zheng Y. 6-alkylsalicylates are selective Tip60 inhibitors and target the acetyl-CoA binding site. Eur J Med Chem. 2012;47(1):337-344.

35. Coffey K, et al. Characterisation of a Tip60 specific inhibitor, NU9056, in prostate cancer. PLoS One. 2012;7(10):e45539.

36. Han Z, et al. Revealing the protein propionylation activity of the histone acetyltransferase MOF (males absent on the first). J Biol Chem. 2018;293(9):3410-3420.

37. Sobreira N, Schiettecatte F, Valle D, Hamosh A GeneMatcher: a matching tool for connecting investigators with an interest in the same gene. Hum Mutat. 2015;36(10):928-930.

38. Firth HV, et al. DECIPHER: Database of Chromosomal Imbalance and Phenotype in Humans Using Ensembl Resources. Am J Hum Genet. 2009;84(4):524-533.

39. Yan $\mathrm{K}$, et al. Deficient histone $\mathrm{H} 3$ propionylation by BRPF1-KAT6 complexes in neurodevelopmental disorders and cancer. Sci Adv. 2020;6(4):eaax0021.

40. Rodríguez S, Eriksson M. Low and high expressing alleles of the LMNA gene: implications for laminopathy disease development. PLoS One. 2011;6(9):e25472.

41. Harel T, et al. Monoallelic and biallelic variants in EMC1 identified in individuals with global developmental delay, hypotonia, scoliosis, and cerebellar atrophy. Am J Hum Genet. 2016;98(3):562-570.

42. Göttlicher M, et al. Valproic acid defines a novel class of HDAC inhibitors inducing differentiation of transformed cells. EMBOJ. 2001;20(24):6969-6978.

43. Conrad T, et al. The MOF chromobarrel domain controls genome-wide H4K16 acetylation and spreading of the MSL complex. Dev Cell. 2012;22(3):610-624.

44. Nielsen PR, et al. Structure of the chromo barrel domain from the MOF acetyltransferase. J Biol Chem. 2005;280(37):32326-32331.

45. Xu P, et al. The NuA4 core complex acetylates nucleosomal histone $\mathrm{H} 4$ through a double recognition mechanism. Mol Cell. 2016;63(6):965-975

46. Smith ER, Cayrou C, Huang R, Lane WS, Côté J, Lucchesi JC. A human protein complex homologous to the Drosophila MSL complex is responsible for the majority of histone $\mathrm{H} 4$ acetylation at lysine 16. Mol Cell Biol. 2005;25(21):9175-9188.

47. Urdinguio RG, et al. Chromatin regulation by Histone $\mathrm{H} 4$ acetylation at Lysine 16 during cell death and differentiation in the myeloid compartment. Nucleic Acids Res. 2019;47(10):5016-5037.

48. Chelmicki T, et al. MOF-associated complexes ensure stem cell identity and Xist repression. Elife. 2014;3:e02024.

49. Sheikh BN, Guhathakurta S, Akhtar A. The non-specific lethal (NSL) complex at the crossroads of transcriptional control and cellular homeostasis. EMBO Rep. 2019;20(7):e47630.

50. Li L, Jin J, Yang XJ. Histone deacetylase 3 governs perinatal cerebral development via neural stem and progenitor cells. iScience. 2019;20:148-167.

51. Koolen DA, et al. Mutations in the chromatin modifier gene KANSL1 cause the 17q21.31 microdeletion syndrome. Nat Genet. 2012;44(6):639-641.

52. Zollino M, et al. Mutations in KANSL1 cause the 17q21.31 microdeletion syndrome phenotype. Nat Genet. 2012;44(6):636-638.

53. Boettger LM, Handsaker RE, Zody MC, McCarroll SA. Structural haplotypes and recent evolution of the human $17 \mathrm{q} 21.31$ region. Nat Genet. 2012;44(8):881-885.

54. Basilicata MF, et al. De novo mutations in MSL3 cause an X-linked syndrome marked by impaired histone $\mathrm{H} 4$ lysine 16 acetylation. Nat Genet. 2018;50(10):1442-1451.

55. Kadlec J, et al. Structural basis for MOF and MSL3 recruitment into the dosage compensation complex by MSL1. Nat Struct Mol Biol. 2011;18(2):142-149.

56. Sadhukhan S, et al. Metabolomics-assisted proteomics identifies succinylation and SIRT5 as important regulators of cardiac function. Proc Natl Acad Sci U S A. 2016;113(16):4320-4325.

57. Ravn K, et al. High incidence of propionic acidemia in greenland is due to a prevalent mutation, 1540insCCC, in the gene for the beta-subunit of propionyl CoA carboxylase. Am J Hum Genet. 2000;67(1):203-206.

58. Pougovkina O, Te Brinke H, Wanders RJ, Houten SM, de Boer VC. Aberrant protein acylation is a common observation in inborn errors of acyl-CoA metabolism. JInherit Metab Dis. 2014;37(5):709-714.

59. Jin Q, et al. Distinct roles of GCN5/PCAFmediated $\mathrm{H} 3 \mathrm{~K} 9 \mathrm{ac}$ and $\mathrm{CBP} / \mathrm{p} 300$-mediated H3K18/27ac in nuclear receptor transactivation. EMBO J. 2011;30(2):249-262.

60. Petrij F, et al. Rubinstein-Taybi syndrome caused by mutations in the transcriptional co-activator CBP. Nature. 1995;376(6538):348-351.

61. Doyon Y, et al. ING tumor suppressor proteins are critical regulators of chromatin acetylation required for genome expression and perpetuation. Mol Cell. 2006;21(1):51-64.

62. Ullah M, et al. Molecular architecture of quartet MOZ/MORF histone acetyltransferase complexes. Mol Cell Biol. 2008;28(22):6828-6843.

63. Lalonde ME, et al. Exchange of associated factors directs a switch in $\mathrm{HBO} 1$ acetyltransferase histone tail specificity. Genes Dev. 2013;27(18):2009-2024.

64. Mattioli F, et al. Mutations in histone acetylase modifier BRPF1 cause an autosomal-dominant form of intellectual disability with associated ptosis. Am J Hum Genet. 2017;100(1):105-116.

65. Suka N, Luo K, Grunstein M. Sir2p and Sas2p opposingly regulate acetylation of yeast histone $\mathrm{H} 4$ lysine16 and spreading of heterochromatin. Nat Genet. 2002;32(3):378-383.

66. Vaquero A, Sternglanz R, Reinberg D. NAD+ -dependent deacetylation of $\mathrm{H} 4$ lysine 16 by class III HDACs. Oncogene. 2007;26(37):5505-5520.

67. Fang J, et al. Sirt7 promotes adipogenesis in the mouse by inhibiting autocatalytic activation of Sirt1. Proc Natl Acad Sci U S A. 2017;114(40):E8352-E8361.

68. Singh SK, Williams CA, Klarmann K, Burkett SS, Keller JR, Oberdoerffer P. Sirt1 ablation promotes stress-induced loss of epigenetic and genomic hematopoietic stem and progenitor cell mainte- 
nance. J Exp Med. 2013;210(5):987-1001.

69. McBurney MW, et al. The mammalian SIR2alpha protein has a role in embryogenesis and gametogenesis. Mol Cell Biol. 2003;23(1):38-54.

70. Orpinell M, et al. The ATAC acetyl transferase complex controls mitotic progression by targeting non-histone substrates. $Е M B O \mathrm{~J}$. 2010;29(14):2381-2394.

71. Hajji N, Wallenborg K, Vlachos P, Füllgrabe J, Hermanson O, Joseph B. Opposing effects of hMOF and SIRT1 on H4K16 acetylation and the sensitivity to the topoisomerase II inhibitor etoposide. Oncogene. 2010;29(15):2192-2204.

72. Norwood J, Franklin JM, Sharma D, D'Mello SR. Histone deacetylase 3 is necessary for proper brain development. J Biol Chem. 2014;289(50):34569-34582.

73. Montgomery RL, Hsieh J, Barbosa AC, Richardson JA, Olson EN. Histone deacetylases 1 and 2 control the progression of neural precursors to neurons during brain development. Proc Natl
Acad Sci U S A. 2009;106(19):7876-7881.

74. Sun B, et al. Regulation of the histone acetyltransferase activity of hMOF via autoacetylation of Lys274. Cell Res. 2011;21(8):1262-1266.

75. Yuan H, et al. MYST protein acetyltransferase activity requires active site lysine autoacetylation. ЕMBO J. 2012;31(1):58-70.

76. Lu L, Li L, Lv X, Wu XS, Liu DP, Liang CC. Modulations of hMOF autoacetylation by SIRT1 regulate hMOF recruitment and activities on the chromatin. Cell Res. 2011;21(8):1182-1195.

77. Nativio R, et al. Dysregulation of the epigenetic landscape of normal aging in Alzheimer's disease. Nat Neurosci. 2018;21(4):497-505.

78. Deciphering Developmental Disorders Study. Large-scale discovery of novel genetic causes of developmental disorders. Nature. 2015;519(7542):223-228.

79. You L, et al. The lysine acetyltransferase activator Brpf1 governs dentate gyrus development through neural stem cells and progenitors. PLoS
Genet. 2015;11(3):e1005034.

80. You L, et al. The chromatin regulator Brpf1 regulates embryo development and cell proliferation. J Biol Chem. 2015;290(18):11349-11364.

81. You L, et al. BRPF1 is essential for development of fetal hematopoietic stem cells. JClin Invest. 2016;126(9):3247-3262.

82. Yan $\mathrm{K}$, et al. The chromatin regulator BRPF3 preferentially activates the HBO1 acetyltransferase but is dispensable for mouse development and survival. J Biol Chem. 2016;291(6):2647-2663.

83. Kim GW, Li L, Ghorbani M, You L, Yang XJ. Mice lacking $\alpha$-tubulin acetyltransferase 1 are viable but display $\alpha$-tubulin acetylation deficiency and dentate gyrus distortion. J Biol Chem. 2013;288(28):20334-20350.

84. Sansregret L, et al. Cut homeobox 1 causes chromosomal instability by promoting bipolar division after cytokinesis failure. Proc Natl Acad Sci U S A. 2011;108(5):1949-1954. 